NBER WORKING PAPER SERIES

\title{
CULTURE: PERSISTENCE AND EVOLUTION
}

\author{
Francesco Giavazzi \\ Ivan Petkov \\ Fabio Schiantarelli \\ Working Paper 20174 \\ http://www.nber.org/papers/w20174 \\ NATIONAL BUREAU OF ECONOMIC RESEARCH \\ 1050 Massachusetts Avenue \\ Cambridge, MA 02138 \\ May 2014
}

We would like to thank Alberto Alesina, Alberto Bisin, Rossella Greco, Luigi Guiso, Claudia Olivetti, John Seater, Andrei Shleifer, Guido Tabellini and participants to the BC Macro Lunch and the NBER Political Economy Program Spring 2014 Meeting, in particular Paola Giuliano, for very useful comments and suggestions. We also thank Julia Schiantarelli for providing inspiration for this paper through her Junior Thesis at Newton North High School, and Hayley Huffman. Finally, we gratefully acknowledge the support of the Italian Ministry for Universities, PRIN grant 2010TBXAXB008. The views expressed herein are those of the authors and do not necessarily reflect the views of the National Bureau of Economic Research.

NBER working papers are circulated for discussion and comment purposes. They have not been peer-reviewed or been subject to the review by the NBER Board of Directors that accompanies official NBER publications.

(C) 2014 by Francesco Giavazzi, Ivan Petkov, and Fabio Schiantarelli. All rights reserved. Short sections of text, not to exceed two paragraphs, may be quoted without explicit permission provided that full credit, including $\odot$ notice, is given to the source. 
Culture: Persistence and Evolution

Francesco Giavazzi, Ivan Petkov, and Fabio Schiantarelli

NBER Working Paper No. 20174

May 2014, Revised August 2016

JEL No. A13,F22,J00,J61,Z1

\section{ABSTRACT}

This paper documents the speed of evolution (or lack thereof) of a range of values and beliefs of different generations of US immigrants, and interprets the evidence in the light of a model of socialization and identity choice. Convergence to the norm differs greatly across cultural attitudes. Moreover, results obtained studying higher generation immigrants differ from those found when the analysis is limited to the second generation and imply a lesser degree of persistence than previously thought. Persistence is also culture specific, in the sense that the country of origin of one's ancestors matters for the pattern of generational convergence.

Francesco Giavazzi

Universita' Bocconi and IGIER

Via Guglielmo Röntgen, 1

Milan 20136

ITALY

and NBER

francesco.giavazzi@unibocconi.it
Fabio Schiantarelli

Department of Economics

Boston College

140 Commonwealth Avenue

Chestnut Hill, MA 02467

and IZA

schianta@bc.edu

Ivan Petkov

Boston College

Department of Economics

140 Commonwealth Avenue

Chestnut Hill, MA 02160

petkovi@bc.edu 


\section{Introduction and Motivation}

Learning how a person's values and beliefs are formed and transmitted from one generation to the next is the first step towards understanding the more general problem of how persistent a society's values and beliefs are - an issue on which there is abundant disagreement. Some contributions argue that values and beliefs are deeply rooted in the country or ethnic group to which a person belongs - being related for example to history or geography — and evolve slowly over time. ${ }^{1}$ Others, instead, suggest that cultural attitudes can change rather quickly in response to changes in economic incentives and opportunities, in technology, and in institutions. ${ }^{2}$ Both views of culture (slow versus fast moving) have truth in them, in the sense that while some cultural traits certainly go back to the distant past and affect today's economic and institutional outcomes, it is also true that many values and beliefs evolve in response to changes in technology, economic environment, and in political institutions.

An important distinction in understanding the process through which a person's values and beliefs are formed is that between "vertical" and "horizontal" transmission. Inside the family, parents shape their children's preferences balancing the desire to share common values with them, with the concern for teaching traits that will make it easier for their children to function in the social environment in which they will live: this is vertical transmission. But children are also exposed to the world outside the family and thus are subject to a process of social imitation and learning external to the family: this is horizontal transmission. ${ }^{3}$ Two different models of cultural transmission are thus at work, as in the models of evolutionary biology ${ }^{4}$. Vertical transmission, like genetic inheritance, tends to be relatively more conservative, giving rise to slow evolution of culture; horizontal transmission, as in an epidemic, may result in a rapid change in the number of people who adopt a new cultural characteristic particularly if it is attractive to the receiver. This can happen, not in historic time, but in the space of a few generations.

Thinking about these issues, immigrants jump out as an ideal group to study. The incentives that give rise to vertical transmission could be particularly strong among immigrants, as early-generations immigrants may want their children to share some of the values that they, or their own parents, brought with them from their country of origin. But some of these inherited values may be at odds with the culture of the new country in which they are living,

\footnotetext{
${ }^{1}$ See Putnam (1993), Guiso, Sapienza and Zingales (2006, 2008, 2013), Tabellini (2008a, 2008b, 2010), Alesina, Giuliano and Nunn (2013), Durante (2009), Roland (2004) and Alesina and Giuliano (2015) for a recent review.

${ }^{2}$ See Gruber and Hungerman (2008), Alesina and Fuchs-Schuendeln (2007), Di Tella, Galiani and Schargrodsky (2007), Giuliano and Spilimbergo (2014), Fernandez (2011), Fehr (2009), and Bowles (1998).

${ }^{3}$ The transmission that occurs from a member of the previous generation who is external to the family to a member of the present generation is often called oblique. We consider it as a part of horizontal transmission.

${ }^{4}$ See Cavalli-Sforza (1981) and (2001, ch.6), Boyd and Richerson (1985, 2005).
} 
possibly hindering productive exchange with other groups, and may be modified by social interactions in the new environment: horizontal transmission could thus also be particularly strong among immigrants.

In this paper we investigate the speed of evolution of a wide range of cultural attitudes for different generations of immigrants to the United States. We look at a variety of attitudes, rather than a single one because we surmise there could be substantial heterogeneity across cultural traits and immigrants' origins in the speed with which attitudes evolve across generations. We study the transmission of attitudes through four generations (a century) because it is possible that some attitudes may appear to be quite persistent within a couple of generations but change significantly by the fourth generation. We use data from the General Social Survey (GSS) to analyze the evolution of cultural attitudes about religion, family, gender, sexuality, cooperation, redistribution, etc., distinguishing between first, second, third and fourth (or higher) generations of British, Irish, German, Italian, Polish, Scandinavian and Mexican immigrants to the United States. The focus on these groups is largely imposed on us by the availability of sufficient data for multiple generations distinguished by country of origin. We use the data contained in 21 waves (the exact number varies across attitudes) of the GSS survey collected between the end of the 1970's and 2014. Although the GSS is far from being perfect, it is the only data source that allows a systematic investigation of the evolution of cultural values for multiple generations, multiple countries of origin and multiple traits.

Immigrants provide a particularly useful laboratory for the study of the evolution of values and beliefs because, as mentioned above, their cultural attitudes are likely to bear the mark of the country from which they, their parents or their grandparents emigrated. ${ }^{5}$ However, they are also influenced by their exposure to US society and its social, political, and economic institutions, often very different from those of the country of origin. They thus provide an interesting quasi-experiment for the effect on inherited cultural attitudes of a change in the economic and social environment. The conditions under which this leads to integration of immigrants or to the emergence of immigration clusters in which separate cultural traits persist has been debated in the theoretical and empirical literature. ${ }^{6}$

In order to provide some structure in discussing the results, we develop a simple model of

\footnotetext{
${ }^{5}$ See Fernandez (2008).

${ }^{6}$ See the seminal paper by Lazear (1999) on the incentives to and conditions for integration in heterogeneous populations and the inter-temporal extension in Konya (2005). Bisin and Verdier (2000), (2001) provide conditions under which heterogeneity in cultural values may be a stable equilibrium in an optimizing model of cultural transmission under imperfect parental empathy. See also Bisin, Topa and Verdier (2004), Tabellini (2008b), and Bisin and Verdier (2010) for a review. See also Guiso, Sapienza and Zingales (2008) for a model of transmission of beliefs, Fernandez (2013) for a model of beliefs formation, and Doepke and Zilibotti (2008) for a model of endogenous preference formation.
} 
socialization and identity choice. The model builds on the contributions by Bisin and Verdier (2001) on the choice of socialization by parents, and on Lazear (1999) and Konya (2005) for a child's choice of her cultural identity. Parents derive utility form the child retaining their original cultural trait, but also consider the possibility that this may hinder the child's ability to interact productively with the majority. The child plays an active role in the model and chooses her identity weighing the expected transaction gains from assimilation and a switching cost that partly depends upon the parents' socialization effort, and which also contains a component that is randomly distributed across the population. Parents choose the optimal level of socialization taking into account the child's optimization problem, knowing the distribution of the switching cost, but not the particular realization for their child. The model yields two possible equilibria: one with complete assimilation and another with the minority group not assimilating. The occurrence or not of assimilation, and its speed when it happens, depend upon a set of parameters that are likely to vary across different cultural traits and across different countries of origin, such as the child's net transaction gains and the switching costs from assimilating, the utility benefit to the parents from the child maintaining the original trait, together with the costs of the socialization effort, and, finally, the discount factor parents apply to the child's utility.

In studying how a person's values and beliefs are formed and transmitted from one generation to the next, and whether or not they converge, we face a number of empirical challenges. First and foremost, immigrants, even from the same country of origin, differ, depending on when the first generation of the "dynasty" they belong to arrived in the US. Irish immigrants who arrived in the 1890s, for example, are clearly different, in terms of the values they brought with them, from post World War II first generation Irish immigrants. One has to account for this in empirical work, in order to separate convergence of values across generations of immigrants from convergence of values over time across countries of origin. We address this problem studying the transmission of values and beliefs within a single dynasty, specifically the one that starts with first generation immigrants born at the end of the 19th century/beginning of the 20th century. We then follow the cohort of the children of this generation, and the cohorts of their grand children and of their grand-grand children.

A second empirical challenge is that, once one allows for a general model which includes generation effects that vary across cohorts for each country, one is left with relatively small cell sizes for the first generation, particularly for some countries. We will address this problem at the end of Section 4, yet one must recognize that the GSS is the only dataset containing information on a range of cultural attitudes for several countries of origin and multiple generations of immigrants within a dynasty. ${ }^{7}$

\footnotetext{
${ }^{7}$ One may wonder whether US Census or CPS data could be used to investigate the convergence of attitudes
} 
We are certainly not the first ones to analyze these issues ${ }^{8}$. However, most existing contributions focus on the persistence of cultural traits for second generation immigrants and on their effect on economic and social outcomes. For instance, Giuliano (2007) presents evidence that cultural heritage is important for living arrangements, Fernandez (2007) for female labor force participation, and Fernandez and Fogli (2009) for female labor force participation and fertility outcomes, all using US census data. Fernandez and Fogli (2006), using the GSS, finds results that are also supportive of an effect of the culture of the country of ancestry on fertility outcomes for US immigrants, although no distinction is made between second and higher generation immigrants. Guiso, Sapienza and Zingales (2006), using the GSS, find evidence suggesting that the trust of US immigrants (not distinguished according to the generation they belong to) strongly depends upon the country of origin. Exceptions, in the sense that they use generations beyond the second, are Antecol (2000) - who finds that culture matters for the gender gap in labor force participation, for both the first, second and higher generations of US immigrants, although less for the latter - and Borjas (1992) who shows that ethnic capital (measured as average ethnic-specific education, professional achievement or wages) has a greater effect on children's education, occupation and wages for both the second and the third generation, although the effect tends to be higher for the second. Algan and Cahuc (2010) show that inherited trust of descendants of immigrants in the US is significantly associated with the level of trust in the country of origin. This results holds even if one limits the analysis to fourth generation immigrants. ${ }^{9}$

The paper has three main findings. Our first result is that time since the original immigration of the ancestors matters: results obtained studying higher generation immigrants differ from those obtained limiting the analysis to the second generation. Thus, finding that the attitudes of second generation immigrants have not converged yet and still closely reflect

over multiple generations. The answer is unfortunately no. When using these data sets one could think, for instance, of focusing on the effect of the country of origin on female labor force participation (an outcome of cultural attitudes about gender roles, in addition to other factors). In the Census or the CPS, however, one can identify, at best, only the birthplace of the respondent and of her parents (available in the Census only up to 1970 and in the CPS from 1971 to 1975). This gives us information on the country of origin of the first and second generation immigrants. In order to identify the birthplace of the ancestors of third or higher generation immigrants, one must rely on self reported ancestry (available in the Census since 1980 and in the CPS since 1994). Note that the periods for which ancestry information is available together with information on the respondent's and her parents' birthplace are not overlapping, making an investigation of convergence across multiple generations (first, second, third and beyond) not possible even for this single outcome.

${ }^{8}$ Earlier contributions in the sociological literature use early waves of the GSS, and focus on the assimilation process of specific groups, such as Italian immigrants in Greeley (1974, ch.4) and Alba (1985, ch.6). The results in Greeley are based on a sample of males only. Both studies emphasize the change, as opposed to the persistence of cultural attitudes, but do not distinguish among different generations.

${ }^{9}$ Rice and Feldman (1997) distinguish the level of civic attitudes for Italian immigrants on the basis of the number of grandparents born in the US and reach the surprising conclusion that the descendants of earlier immigrants are more likely to give less civic responses than the descendants of later immigrants. 
those of the country of origin, does not imply per se that attitudes are very persistent. For instance, we find that the beliefs that shape trust of second generation immigrant towards other members of society remain different from the prevailing US norm and still bear strongly the mark of the country of origin. However, such differences become smaller when one considers fourth or higher-generation immigrants.

Second, we provide evidence of heterogeneity across cultural traits in the speed with which they evolve across generations and the degree to which converge to the prevailing norm. We find, for instance, that attitudes towards cooperation (the trustworthiness, helpfulness and fairness of others) display the highest degree of convergence by the fourth generation, as successive generations adapt to the norms of the new society in which they live. Attitudes towards politics and the role of government, sexual morality and abortion exhibit the lowest degree of convergence, followed by religious attitudes. Attitudes towards gender roles occupy an intermediate position, with attitudes towards the role of women in the labor market converging faster than those related to the role of women in politics. Family attitudes also display on average an intermediate level of convergence, but there is substantial heterogeneity among them.

Many of these results are largely consistent with one prediction of our simple model: faster convergence is observed for attitudes that are likely to generate larger transaction gains from assimilation, such as attitudes towards cooperation. Convergence is also slower for attitudes for which the utility gain to the parents from the child retaining the original trait (or the cost for the child to abandon them) is likely to be higher, such as some moral and religious values and political orientation .

Third, we find that persistence is "culture-specific" in the sense that the country from which one's ancestors came from matters in defining the pattern of integration (or lack thereof) with respect to the entire set of cultural traits. Moreover, the strength of the family in each country of ancestry and the degree of difficulty in learning English are (negatively) correlated with the fraction of attitudes for which we observe faster convergence. These results too could be interpreted in the light of our model: switching costs, for instance, are likely to be related to language proximity and to the strength of family ties. However, given the small number of countries in our sample, this results must be taken with a grain of salt.

The plan of the paper is as follows. In Section 2 we illustrate a simple model of parents' socialization and children's identity choice. In Section 3 we discuss how we measure cultural attitudes in the GSS, how we define generations and ethnic origin and which countries (or groups of countries) we use in our analysis. In Section 4 we describe how we recover the country of origin effect for different generations, dynasties and time periods, while in Section 5 we illustrate our measure of cultural "convergence". In Section 6 we present and discuss our 
main empirical results. Section 7 contains several robustness checks and extensions. Section 8 concludes.

\section{Why Persistence Can Differ Among Cultural Traits and Countries of Origin : A Model of Cultural Trans- mission}

This section contains a simple model that will help interpret our main empirical findings, namely that different cultural traits may converge at varying speed, or not converge at all. Moreover, the dynamics of cultural convergence may differ across cultures i.e. in terms of our empirical work, across countries of origin. The model is based on the idea that a person's traits evolve through two parallel processes: vertical transmission within the family and horizontal transmission associated with social interactions outside the family. The model draws on the vast literature carefully reviewed in Bisin and Verdier (2010). ${ }^{10}$

The model is set up as follows. Assume there is one cultural trait in the population that can take two values: one associated with the minority, denoted by $m$ and the other associated with the majority, denoted by $M$. Think of the two traits as representing, for instance, the attitude towards pre-marital sex, one of the attitudes whose evolution we study in our empirical analysis. Recent immigrants (the minority) might still carry their cultural attitudes of the country of origin, which could be quite different from those of the majority in the United States, the new social environment in which they live.

We normalize the population to 1 and assume that the initial size of the minority is $q$. Consider a second-generation immigrant belonging to the minority group. Personal attitudes are shaped by two forces: "vertical" transmission within the family and "horizontal" transmission from social interactions outside the family. Traits are first transmitted inside the family from parents to their children. As children interact with people outside the family, they may realize that the traits acquired from their parents are not ideal (in a sense that we shall make precise in a moment) for social interactions outside the family. For instance, if the norm in society (the norm of the majority) is that young people live together before deciding whether or not to get married, excluding pre-marital sex will make it more difficult for the child to find a partner and get married. However, breaking with a more traditional view of sexual morality may also generate a costly conflict with one's family, the more so the greater the parents' effort to educate the child.

We shall proceed in three steps. First we study the child's identity choice problem: what

\footnotetext{
${ }^{10}$ See also Pichler (2010), Vaughan (2013), and Panebianco (2014).
} 
determines her decision whether or not to "assimilate", that is to abandon the minority trait and acquire the majority trait. ${ }^{11}$ Building on Lazear (1999) and Konya (2005), we assume that switching from the old to the new trait allows a minority member to interact more productively with the majority. However, it also generates a transaction cost in dealing with members of the minority. Moreover, abandoning the original family trait implies a utility cost for the child that, in part, depends upon the effort the parents have put in socializing her. Then we shall go back and analyze the parent's socialization problem: parents prefer children with their own cultural trait and hence educate them to this trait, as in Bisin and Verdier (2001). The parent however also "empathizes" with her child, in the sense that she understands that the trait she is trying to transmit may hinder the child's opportunities in the new society. Her educational decision will balance these two incentives.

To keep the problem simple, we assume that each individual lives two periods. In the first period she is socialized to the family's values by her parents and interacts with the other young people in society. In the second period she becomes the single parent of a child and decides how much effort to put in socializing the child to her own trait - for instance spending time teaching her ancestors' values. Finally, having analyzed the child's decision whether or not to assimilate, given the education effort optimally chosen by her parent, we shall study how the size of the minority evolves over time, given that the cost of assimilation is distributed randomly in the population.

We show that there are two possible equilibria: one in which no child assimilates and the size of the minority group remains constant at the initial level, and one in which instead children assimilate and the minority trait eventually disappears from society. Which of these two equilibria occurs and the speed of convergence to the full assimilation equilibrium depend upon a set of parameters that capture the cost and benefits of assimilation for the child and of the socialization effort for the parent, and that are likely to vary across cultural traits, and also across countries of origin.

\subsection{The Child's Identity Choice Problem}

The child's problem is a simple variant of Lazear $(1999)^{12}: V^{i},(i=m$ or $M)$ denotes the surplus produced by a social interaction between two persons both belonging to the same group - minority or majority. We assume that the two surpluses are identical $\left(V^{m}=V^{M}=\right.$ $V$ ), a simplifying assumption which is irrelevant for our results. The interaction between two persons with different cultural traits implies a loss. More specifically, $V\left(1-\theta^{M}\right)$ is the surplus

\footnotetext{
${ }^{11}$ See also the seminal paper on identity choice by Akerlof and Kranton (2000), as well as Bisin, Pattachini, Verdier, and Zenou (2011).

${ }^{12}$ See Konya (2005) for a dynamic extension.
} 
produced by a social interaction between a person, whose parents belong to the minority and who has not assimilated, with another person belonging to the majority, with $0<\theta^{M}<1$. $V\left(1-\theta^{m}\right)$ is the surplus of the interaction between a person whose parents belong to the minority and who has acquired the majority trait, with another person from the minority, with $0<\theta^{m}<1$. We will assume that $\theta^{M}>\theta^{m}$ because it is plausible that the child of a minority parent retains some ability to interact with members of the minority even if she assimilates. There is no loss in the transaction when two people have the same trait, that is in this case the surplus is $V$. The proportion of the minority group in the population is $q<\frac{1}{2}$ (we omit the time subscript here to keep the notation light). $d\left(\tau, z_{i}\right)$ is the utility cost for a member of the minority for abandoning the parent's trait: it is increasing with the parent's socialization effort $\tau$ and also includes an additive person specific stochastic component $z_{i}$ that can be interpreted as the cost of learning the new (majority) trait for individual $i$, so that $d\left(\tau, z_{i}\right)=d(\tau)+z_{i}$, with $d(\tau)^{\prime}>0$. We assume $z_{i}$ to be distributed randomly in the population according to the distribution function $G($.$) . The child knows z_{i}$, while the parent does not observe it, but knows its distribution $G($.$) .$

The child meets at random individuals from the minority or majority groups with probability $q$ and $1-q$ respectively. Following Lazear (1999) we assume that the child decides whether or not to assimilate at the beginning of the period, knowing the probability of meeting a minority or a majority member, but before having actually met them. Her expected utility is therefore equal to $q V+(1-q)\left(1-\theta^{M}\right) V$ when the child does not assimilate, and to $q\left(1-\theta^{m}\right) V+(1-q) V-d(\tau)-z_{i}$ when she assimilates. Children are myopic, in the sense that they do not look ahead to when they will become parents. A child $i$ assimilates if the expected gain from assimilation is higher than the expected gain from non-assimilation:

$$
(1-q) V \theta^{M}-q \theta^{m} V-d(\tau)-z_{i} \geq 0
$$

Defining the cumulative density of $z_{i}$, with support $[\underline{z}, \bar{z}]$, the proportion of minority individuals that assimilate after a draw of $z_{i}$ is given by:

$$
G\left((1-q) V \theta^{M}-q \theta^{m} V-d(\tau)\right)
$$

If $(1-q) V \theta^{M}-q \theta^{m} V-d(\tau)>\bar{z}$ the child will always decide to assimilate $(G()=1$.$) .$ If $(1-q) V \theta^{M}-q \theta^{m} V-d(\tau)<\underline{z}$ the child will never assimilate $(G()=0$.$) . When \underline{z} \leq$ $(1-q) V \theta^{M}-q \theta^{m} V-d(\tau) \leq \bar{z}$, the child will assimilate with some probability. Assume for simplicity that $z_{i}$ is uniformly distributed on $[\underline{z}, \bar{z}]$. In this case the probability of assimilation 
and the proportion of minority individuals who assimilate is given by:

$$
\operatorname{Prob}\left(z_{i} \leq(1-q) V \theta^{M}-q \theta^{m} V-d(\tau)\right)=\int_{\underline{z}}^{(1-q) V \theta^{M}-q \theta^{m} V-d(\tau)} \frac{1}{\bar{z}-\underline{z}} d t=\frac{(1-q) V \theta^{M}-q \theta^{m} V-d(\tau)}{\bar{z}-\underline{z}}
$$

\subsection{The Parent's Socialization Problem}

Each family is a single-parent family and produces only one child. As in Bisin and Verdier (2001) the parent can socialize the child at a cost $c(\tau)$, increasing in $\tau$, and she derives utility $\varphi(\tau)$ if the child maintains the family trait, which occurs with a probability she can affect through her educational effort. The parent also cares about her child's utility and how it is affected by her actions that contribute to determining, through $d(\tau)$, the probability of assimilation, and, hence, how productively the child will relate with the majority (and the minority). The extent of empathy is described by $\beta$ : for $\beta=0$ the parent doesn't care about the child's utility and only cares about her wish that the child does not assimilate. We abstract from all components of the parent's utility that do not depend upon the costs and benefits of educating the child. Finally we also assume that the parent only cares about her immediate descendants.

Thus the parent maximizes her expected utility $w(\tau)$ given by:

$$
\begin{aligned}
w(\tau)= & -c(\tau)+\varphi(\tau) \operatorname{Prob}(\text { no child assimilation })+ \\
& +\beta \operatorname{Prob}\left(\text { no child assimilation) }\left[q V+(1-q) V\left(1-\theta^{M}\right)\right]\right. \\
& +\beta \operatorname{Prob}(\text { child assimilation })\left[q\left(1-\theta^{m}\right) V+(1-q) V-d(\tau)\right]- \\
& -\beta \int_{\underline{z}}^{(1-q) \theta^{M} V-q \theta^{m} V-d(\tau)} \frac{z_{i}}{\bar{z}-\underline{z}} d z_{i}
\end{aligned}
$$

Let us assume that $c(\tau)=\frac{c}{2} \tau^{2}, \varphi(\tau)=\varphi_{0}$, and $d(\tau)=d \tau{ }^{13}$ The parent's optimal socialization effort is determined by the following first order condition:

$$
c \tau+\beta d \frac{(1-q) \theta^{M} V-q \theta^{m}-d \tau-\underline{z}}{\bar{z}-\underline{z}}=\frac{\varphi_{0} d}{\bar{z}-\underline{z}}
$$

The interpretation is simple: the left hand side is the marginal cost to the parent from varying $\tau$, composed by the marginal direct socialization/education cost and by the expected change in the assimilation cost for the child, discounted by $\beta$ (the parent's imperfect empathy

\footnotetext{
${ }^{13}$ We could allow $\varphi(\tau)$ to equal $\varphi_{0}+\varphi_{1} \tau$ with $\varphi_{1}>0$, but this would complicate the algebra without improving the intuition.
} 
parameter); the right hand side is the change in the expected direct benefit for the parent from non-assimilation. Solving for the optimal level of $\tau, \tau^{*}$, one obtains:

$$
\tau^{*}=\frac{\varphi_{0}-\beta\left[(1-q) \theta^{M} V-q \theta^{m} V-\underline{z}\right]}{\frac{c(\bar{z}-\underline{z})}{d}-\beta d}
$$

For concavity of the objective function $\frac{\partial^{2} w}{\partial \tau^{2}}=-c+\frac{\beta d^{2}}{\bar{z}-\underline{z}}<0$ and hence the denominator in (6) is positive. We assume that $\varphi_{0}-\beta\left[(1-q) \theta^{M} V-q \theta^{m}-\underline{z}\right] \geqslant 0$ to guarantee that the parent's effort is non negative. The comparative static for $\tau^{*}$ is intuitive. The parent's effort is increasing in $\varphi_{0}$, her benefit if the child does not assimilate. It is instead decreasing in $c$, the cost of the effort put into educating the child. It is also increasing in $\theta^{M}$, the penalty for the descendant of a minority parent in interacting with members of the majority, if she holds on to the family trait, and decreasing in $\theta^{m}$, the penalty for the descendant of a minority parent in interacting with members of the minority, if she adopts the majority trait. In the former case the benefit of assimilating for the child increases, while in the latter it decreases. A strong educational effort by the parent is thus a hindrance for the child, the more so the larger is $\theta^{M}$ and the smaller is $\theta^{m}$. The empathic parent internalizes this and reduces her socialization effort the larger is $\theta^{M}$ and increases it the smaller is $\theta^{m}$.

For given values of $\theta^{M}$ and $\theta^{m}$, an increase in $q$ has a positive effect on the parent's socialization effort because it decreases the probability of meeting a member of the majority, diminishing the expected penalty for descendants of minority parents associated with interacting with the majority (when not assimilated) and increases the cost of interacting with members of the minority (when assimilated). Note that our model does not display the "cultural substitutability property" of Bisin and Verdier (2001), whereby a minority parent makes a greater effort at socialization when $q$ is small.

The effect on the parent's socialization effort of an increase in the total surplus from transactions is negative, as we have assumed that $q<\frac{1}{2}$ and $\theta^{M}>\theta^{m}$, so that the transaction net gains from assimilation are positive and the (partly) empathic parent takes this into account, therefore reducing $\tau^{*}$. The effect of the parameter $d$, that captures the cost for the child of assimilating, and that depends on the parent's educational effort, is positive: the higher is $d$, the more effective is the socialization technology and this induces the parent to use it more intensely (increasing her effort). The effect of the discount factor $\beta$ is ambiguous and the reason is simple: if $\beta$ increases, it means that the parent gives more weight both the the child's net transaction benefits of assimilation $\left((1-q) \theta^{M} V-q \theta^{m}\right)$ and to the switching cost of assimilation $(d \tau)$. The first effect leads the partly emphatic parent to decrease $\tau^{*}$, so that the child can reap those benefits; the second leads to an increase in $\tau^{*}$. Hence the effect of $\beta$ is ambiguous. Finally, for a given spread of the distribution, $\bar{z}-\underline{z}$, a decrease in 
$\underline{z}$, which generates a leftward shift of the distribution, decreasing its mean, but keeping the variance constant, is associated to a decrease in $\tau^{* 14}$ : again, this is because the probability of assimilation increases, which increases the penalty for the child of dropping the family trait, a penalty that is greater the larger the parent's educational effort. Given $\underline{z}$, an increase in $\bar{z}-\underline{z}$ has the opposite effect by a similar logic.

\subsection{Assimilation and Non-Assimilation Equilibria and Dynamics}

Let us assume that $\underline{z} \leq(1-q(0)) \theta^{M} V-q(0) \theta^{m} V-d \tau^{*} \leq \bar{z}$, where $q(0)$ is the initial proportion of the minority group in the population, so that there is an incentive to assimilate for at least some members of the minority. In this case the probability of assimilation evaluated at the optimal parent's effort, $\tau^{*}$, is ${ }^{15}$ :

$$
G\left(\left(1-q_{t}\right) \theta^{M} V-q \theta^{m} V-d\left(\frac{\varphi_{0}-\beta\left[\left(1-q_{t}\right) \theta^{M} V-q_{t} \theta^{m}-\underline{z}\right]}{\frac{c(\bar{z}-\underline{z})}{d}-\beta d}\right)\right)
$$

This is also the proportion of minority members in the population that assimilate. It is easy to see that this proportion is unambiguously increasing in $V$ and $\theta^{M}$, and decreasing in $d$, $\theta^{m}$ and $q$. This is the result of the direct effect of these parameters on $G$ (.) and their effect through $\tau^{*}$. The effect of the remaining parameters mimics the effect on $\tau^{*}$ with the opposite sign: the proportion of minority members that assimilates, increases in $c$ and decreases in $\varphi_{0}$; the effect of the discount factor $\beta$ is again ambiguous; for a given spread of the distribution, $\bar{z}-\underline{z}$, a decrease in $\underline{z}$, which generates a leftward shift of the distribution, decreasing its mean, but keeping the variance constant, is associated with an increase in $G($.$) ; given \underline{z}$, an increase in $\bar{z}-\underline{z}$, instead, decreases $G($.$) .$

The decrease in the proportion of the minority between $t+1$ and $t,-\left(q_{t+1}-q_{t}\right)$ equals the proportion of the minority that assimilates between these two dates $G\left(\left(1-q_{t}\right) \theta^{M} V-q_{t} \theta^{m} V-d \tau_{t}^{*}\right)$, times the size of the minority at $t, q_{t}^{16}$ :

$$
\begin{aligned}
q_{t+1}-q_{t} & =-G\left(\left(1-q_{t}\right) \theta^{M} V-q_{t} \theta^{m} V-d \tau_{t}^{*}\right) q_{t} \\
& =-\frac{\left(1-q_{t}\right) \theta^{M} V-q_{t} \theta^{m} V-d \tau_{t}^{*}-\underline{z}}{\bar{z}-\underline{z}} q_{t}
\end{aligned}
$$

with $\tau_{t}^{*}$ defined in (6). Equation (8) represents the dynamics of the system when $\underline{z} \leq$

\footnotetext{
${ }^{14}$ Recall that the mean of the uniform distribution is $\frac{\bar{t}+\underline{t}}{2}$, while the variance is $\frac{(\bar{t}-\underline{t})^{2}}{12}$.

${ }^{15}$ If $(1-q(0)) V \theta^{M}-q(0) \theta^{m} V-d \tau^{*}>\bar{t}$, the model would generate an uninteresting and implausible dynamics with instantaneous full assimilation.

${ }^{16}$ Assuming that no member of the majority acquires the minority trait is equivalent to assuming that $q \theta^{m, M} V-(1-q) \theta^{M, M} V-d^{M} \tau^{M}<\underline{\mathrm{t}}^{M}$, where the superscript $M$ (second superscript for the $\theta$ parameter) denotes the parameters for the majority. In other terms, for all members of the majority, the gain from more efficient transactions is exceeded by the combined costs of acquiring the minority trait.
} 
$\left(1-q_{t}\right) \theta^{M} V-q_{t} \theta^{m} V-d \tau_{t}^{*} \leq \bar{z}$. When $\left(1-q_{t}\right) \theta^{M} V-q \theta_{t}^{m} V-d \tau^{*} \leq \underline{z}$ nobody assimilates, $G()=$.0 and $q_{t+1}-q_{t}=0$. This observation allows us to determine the possible steady state equilibria (where $q_{t+1}-q_{t}=0$ ) and their stability properties. Consider first the value of $q_{t}$, $\tilde{q}$, such that $(1-\tilde{q}) \theta^{M} V-\tilde{q} \theta^{m} V-d \tau *=\underline{z}$ so that there is no gain from assimilation. For greater (smaller) values of $q$ the net gain is negative (positive). It is easy to show that (see Appendix 2, available online, for details on the dynamics and on the steady-state equilibria):

$$
\tilde{q}=\frac{\theta^{M} V-\frac{\varphi d^{2}}{c(\bar{z}-\underline{z})}-\underline{z}}{\theta^{M} V+\theta^{m} V}
$$

Moreover, $0<\tilde{q}<1$. If $\widetilde{q}<q_{0}<\frac{1}{2}$, then the initial proportion of the minority is an equilibrium because there is no net gain from assimilation. Recall that the equation of motion assumes that no member of the majority adopts the minority trait, which is reasonable if indeed we are dealing with a minority $\left(q_{0}<\frac{1}{2}\right)$. If $q_{0}<\operatorname{Min}\left(\frac{1}{2}, \tilde{q}\right)$, the steady state equilibrium implies full integration $(q=0)$. The full integration equilibrium is locally stable with the minority in this case gradually shrinking in size. All this is summarized in Figure 1a and Figure 1b, where the steady state(s) and dynamics of the system are represented. The phase line is upward-sloping and convex and it intersects the 45 degree line at 0 and $\tilde{q}$. In Figure 1a we present the phase diagram for the case in which $\widetilde{q}<\frac{1}{2}$, so that two types of equilibria exist, one with full integration and one with no integration (associated, for instance, with an initial size of the minority equal to $q_{0}^{a}$ and $q_{0}^{n a}$ respectively). In Figure 1b, we present the case in which $\tilde{q} \geq \frac{1}{2}$ so that only the full integration equilibrium exists. Finally, it is easy to see that $\tilde{q}$ increases and hence the range of initial values of $q_{0}$ for which the full assimilation equilibrium occurs becomes larger with the loss for a non assimilated person in her dealing with the majority, $\theta^{M}$, with the size of the total surplus from the transaction, $V$, with the cost to the parents for the socialization effort, $c$, with an increase in $\bar{z}-\underline{z}$ for a given $\underline{z}$ (so that both its mean and variance increase). $\tilde{q}$ instead decreases with the penalty for an assimilated child of a minority parent from dealing with members of the minority, $\theta^{m}$, with the effectiveness of the socialization technology, $d$, with the direct benefit to the parent of the child maintaining the original trait, $\varphi_{0}$, and with a shift to the right of the distribution of $z_{i}$ (so that the mean increases for a given spread of the distribution). Note that the parent's discount factor, $\beta$, has no effect on $\tilde{q}$. This is because at $q=\tilde{q}$, the probability of assimilation is zero, so the second term on the left hand side of the first order condition for $\tau$, equation (5), is zero, i.e. there is no expected cost for the parent from the child assimilating. As a result, at $q=\tilde{q}, \beta$ does not matter for $\tau^{*}$ and, hence, for $\tilde{q} \cdot{ }^{17}$

\footnotetext{
${ }^{17}$ In the model the decision whether or not to assimilate is along a single dimension, that is a single attitude. The results however directly extend to the contemporaneous choice of more than one trait, provided
} 
Summarizing, our simple model can help us think about the different speed of convergence of various attitudes, as they are shaped by vertical and horizontal transmission. Cultural attitudes differ in the advantage that assimilation confers to the child in transacting with the majority and in the costs that assimilation implies for her, partly shaped by the parent's socialization effort. They also differ in the utility gain they imply for the parent when a child retains the minority cultural trait and in the cost that the parent's educational effort entails. For attitudes related to cooperation, such as trust and views of other being helpful and fair, there are likely to be large transaction gains for the child from assimilating. Trust, for instance, plays an important role in economic and social interactions and one can easily imagine, how, for instance, it may pay for an individual to trust others, even if starting from a relatively low trust level. Indeed, in the context of repeated interactions, the gain from defecting from the norm is likely to be short lived. For other traits, such as such as those related to moral values concerning abortion or sexuality, religious attitudes, general political views, and some family or gender attitudes, the transaction payoff from converging to the majority trait is likely to be smaller. Moreover, for such attitudes there may be large gains for the parents if the child maintains the minority trait, or a large cost for the child if she abandons her family's traditional values and beliefs. The model also suggests that patterns of integration may differ depending on the country of origin of each immigrant group because of cross country variation, for each cultural attitude, in the costs and benefits of integration. For instance, cross country variation in the strength of family ties may be reflected in differences in the perceived benefit for the parents from the child not dropping the trait transmitted within the family. Similarly, the cost for the child of acquiring a new trait may differ across countries. We will use these insights in discussing the empirical evidence on the heterogeneity across attitudes in the speed of convergence of values and beliefs of successive generations of immigrants to the US, and how it varies across countries of origin.

we exclude interactions across attitudes. Assume there are two traits $a=1$, 2 , each one of them dichotomous, as we have assumed so far. Assume that costs and benefits are additive and that there is no interaction between the two trais, that is socialization $c\left(\tau_{1}\right)+c\left(\tau_{2}\right)$ costs for the parents are and direct socialization benefits are $\varphi\left(\tau_{1}\right)+\varphi\left(\tau_{2}\right)$. Assume that switching costs are also additive for the child, $d\left(\tau_{1}\right)+d\left(\tau_{2}\right)$, and, to avoid multivariate distributions, that the two stochastic terms $z_{1}$ and $z_{2}$ are independent. Finally assume that the net benefits associated with each attitude are $\theta_{a}^{M *}\left(1-q_{a}\right) V_{a}-\theta_{a}^{m *} q_{a} V_{a}-d\left(\tau_{a}\right)-z_{a}, a=1,2$ again assuming lack of interaction. In this simple case the conditions for $\tau_{1}^{*}$ and $\tau_{2}^{*}$ are identical to those we have derived and simply need to be indexed by $a=1,2$. Of course the model would be more complicated if we allowed for cross affects across attitudes, but this is not central to our paper and we leave this extension for future research. 


\section{Measuring Cultural Attitudes and Defining Genera- tions and Country of Origin in the GSS}

Our measurement of cultural attitudes is based on the General Social Survey (GSS). We use multiple (22) waves of the GSS, starting in 1978 and ending in 2014. Each wave includes a core set of questions that remains in the survey in each year in which it was conducted. This core includes personal information such as age, income, region of residence and family origin, as well as information on personal views on a variety of topics such as family values, gender roles, religious beliefs, sexual behavior, cooperation, role of government, etc.

One of the advantages of the GSS is that it allows us to analyze a wide variety of attitudes over several generations of immigrants. We have selected the attitudes for which data were available over a relatively long span of time, up to three decades (or slightly more). For ease of interpretation we have grouped attitudes (or questions) into several broad categories. ${ }^{18}$ The list of categories, variables, and coding choices is provided in Table 1. Group A deals with views on cooperation and social interactions. It includes questions about trustworthiness (trust), fairness (fair) and helpfulness of others (helpful). Group B includes attitudes towards government intervention - should the government redistribute income (eqwlth), or provide a safety-net for the poor (helppoor) - and overall political views (polviews). Group C surveys different religious attitudes such as the frequency of attendance to religious services (attend), the frequency of personal prayer (pray), the strength of affiliation with one's religion (reliten), the belief in afterlife (postlife) and the approval of prayer in public schools (prayer). Group D includes attitudes about family and children. Questions in this group elicit views on the degree of parental consent in teenage access to birth control (pillok), on the restrictiveness of divorce law (divlaw), on the co-residence of multiple generations (aged) - i.e. whether one approves of children living with their parents beyond a certain age, and on the frequency of evenings spent with relatives (socrel). Furthermore, this group also includes views on preferred qualities in children such as obedience (obey) and independence (thnkself). Group E surveys views on gender roles. Participants in the GSS are asked to express their opinion concerning various statements describing the role of women in the labor market, in politics and at home: should a woman work even if the husband can support her (fework)?; can working mothers have a warm relationship with their children (fechild)?; women should take care of running the home while men run the country (fehome); women are not suited for politics (fepol). Group F reports views on legalized abortion for any reason (abany) or restricted to cases of risk for the mother's health, defects in the fetus, or rape (abrisk). Group G covers

\footnotetext{
${ }^{18}$ For the choice of groups, we have followed one of the available codebooks for the GSS. See Muennig, Kim, Smith, and Rosen (2011).
} 
attitudes towards sexual behavior such as pre-marital sex (premarsx) and homosexual sex (homosex). Group H is a residual group: it contains views on whether social mobility is the result of hard work versus help or luck (getahead), a belief that could not be easily classified in any of the other groups.

The premise of our study is that values and beliefs are formed in part as a result of one's upbringing, and in part through the influence of factors external to the family such as peers, institutions, and economic circumstances. Consequently, values and beliefs depend both on the country of origin of a person's ancestors, as well as on her generation (to be defined below). The country of origin is an important determinant of culture as it encodes the history of a people, encompassing past technological, economic, institutional and cultural environments. The generation of a person is important given that the temporal "distance" from the country of ancestry may be associated with a dilution of the original cultural trait through longer exposure to a different set of economic and social opportunities, to different institutions, and cultural influences.

We consider the evolution of attitudes over multiple generations (up to the fourth). As a result, we are constrained by data availability to focus on immigrants to the US from a limited number of European countries and from Mexico. We focus on countries for which we have relatively numerous observations: Great Britain (GB), comprising England, Wales and Scotland, Germany, (GER), Poland (POL), Ireland (IRE), Italy (ITA) and Mexico (MEX). In addition we consider Scandinavian immigrants from Denmark, Norway, Sweden and Finland as a single group (SCA) on the basis of a relatively common cultural background. ${ }^{19}$

We define the generation to which an immigrant belongs following what is typically assumed in this literature. We define a person to be a first-generation immigrant if he/she was born outside of the United States. Immigrants are defined to be second-generation if they are born in the US and at least one of their parents was born abroad, and third-generation if they are born in the US, all of their parents are born in the US and at least two of their grandparents were born abroad. Lastly, a person is said to be of fourth-generation-or-more if he/she is born in the US, all his/her parents are born in the US and at most one grandparent was born abroad. ${ }^{20}$ With this definition the last category includes fourth generation immigrants as well as people of a higher generation who still declare a specific European country of origin. In defining the country of origin we use the answer to the question: "From what countries or part of the world did your ancestors come?". If more than one country is indicated, the respondent is asked: "Which one of these countries do you feel closer to?". $79 \%$ percent of the sample can identify a main country of origin affiliation. The definition could, in principle,

\footnotetext{
${ }^{19}$ For other Southern and Eastern European countries and for the French we do not have enough observations to reliably estimate country-generation-cohort specific effects.

${ }^{20}$ See Algan and Cahuc (2010).
} 
be made tighter by limiting our analysis to respondents who indicate only one country. This, however, would reduce substantially the number of observations, as only $50 \%$ percent of the sample chooses just one country. Therefore we will not pursue this option here.

\section{Recovering Country of Origin Effects for Different Generations within a Single Dynasty}

The way an individual perceives the world is shaped by the values and beliefs of his/her parents. The attitudes of one's parents are, in turn, shaped by their parents. This implies that an individual's ancestral origin is an important factor determining his/her values and beliefs. In order to capture the extent to which someone's country of origin impacts his/her attitudes, we estimate a Probit model which includes indicator variables for one's ancestry. ${ }^{21}$ We allow the effect of ancestry to depend upon the temporal "distance" from the country of origin. This distance is measured by whether the immigrant is first, second, third, or fourth or higher generation. Moreover the ancestry effect will depend upon the birth cohort of an individual, since the cultural heritage brought by immigrants and transmitted to their descendants depends upon when they left the mother country and came to the US (we will also assume a 25 year interval between cohorts). We allow the effect of the country of origin to depend on generation and cohort in a multiplicative fashion, imposing as little restrictions as possible on the data. We will use these effects to chart the evolution of attitudes within the only complete "dynasty" we observe in our sample. More precisely, we estimate the following Probit model:

$$
\operatorname{Pr}\left(y_{t}^{i}=1\right)=\sum_{o \in O} \sum_{g \in G} \sum_{c \in C} \beta_{o, g, c}\left(I_{\left(\text {Origin }^{i}=o\right)} \times I_{\left(\text {Generation }^{i}=g\right)} \times I_{\left(\text {Cohort }^{i}=c\right)}\right)+\theta X_{t}^{i}
$$

where $y_{t}^{i}$ takes the value of 1 if a certain event has occurred for individual $i$ in wave $t$. $I_{(\text {. . ) are }}$ indicator functions that take the value of 1 if the condition in the subscript is satisfied, 0 otherwise. The sums are defined over three different sets: set $O$ includes all possible countries of origin as defined in Table 2; set $G$ includes each of the four possible generations of immigrants; set $C$ includes four groups of respondents - those born in the periods 1892-1916, 1917-1941, 1942-1966 and after 1967. The set of controls includes: income, education, mother's education, father's education, age, age ${ }^{2}$, year-of-the-survey dummy, gender, number of children, marital status, work status, religion, regional indicators, and urbanization indicators. Clearly variables such as income and education may be related to the country of origin: immigrants

\footnotetext{
${ }^{21}$ Responses to each of the GSS questions are re-coded to produce a binary outcome (see Table 1).
} 
and descendants of people from different countries of origin, may, for instance, attribute different importance to education. Yet, we prefer to define country of origin effects net of these factors, in an attempt to capture deeper cultural values and beliefs that go beyond personal characteristics and circumstances. ${ }^{22}$ Consequently, the evolution of attitudes that this paper analyzes is not explained by changes in the level of education or income of immigrants over time. These individual controls are held constant when we compare changes of attitudes across different immigrants. Finally, note that we include survey-year effect common to all respondents to capture general variations of attitudes over time. Summarizing, the countrygeneration effect is based on the estimated value of $\beta_{o, g, c}$ with $o \in\{1, . ., 7\} ; g \in\{1, . ., 4\}$; $c \in\{1892-1916,1917-1941,1942-1966, \geqslant 1967\}$.

Our sample includes responses of immigrants whose ancestors moved to the U.S. during different periods. For example, the ancestors of some of our respondents arrived with the large migration waves around the turn of the twentieth century, while the ancestors of others immigrated more recently. In order to avoid mixing dynasties of immigrants that started at different points in time, and hence brought with them different attitudes, in our empirical work we focus on the four generations of the only full dynasty of immigrants observable in our data - the one that starts with the first generation arriving between 1892 and 1916 and ending with the last generation being born after 1967. The attitudes of the first generation of this dynasty is captured by $\beta_{o, 1,1892-1916}$, those of the second generation by $\beta_{o, 2,1917-1941}$, those of the third generation by $\beta_{o, 3,1942-1966}$, and, finally, those of the fourth generation (or higher) by $\beta_{o, 4, \geqslant 1967}$. For some countries the GSS does not have many respondents who are both first generation and belong to the cohort of 1892-1916. In order to have enough observations for the first generation of each country, we assume that the first generation of the 1892-1916 cohort and of the 1917-1941 cohort are characterized by the same coefficient $\left(\beta_{o, 1,1892-1916}=\beta_{o, 1,1917-41}\right)$. In Table 3, Part 1 , we report, as an example, the number of observations for each country, generation and cohort for the respondents to the question about trust. In Part 2 of the table we summarize the number of observations available to identify the country-generation-cohort effects for the 1892-1916 dynasty (allowing for the effects of the first generation of the 1892-1916 and 1917-1941 cohorts to be identical)

\section{$5 \quad$ Measuring Convergence in Cultural Attitudes}

In this section we illustrate how we measure and assess whether or not there is convergence in the cultural attitudes of different generations of immigrants towards the norm set by the

\footnotetext{
${ }^{22}$ See also Algan and Cahuc $(2007,2010)$ and Giavazzi, Schiantarelli, and Serafinelli (2013). In our robustness section we also experiment with a more minimalist list of controls, including only age, age squared, year of the survey, gender, religion, regional indicators, and urbanization indicators.
} 
more established and dominant groups. We start by calculating the deviation of the attitude of a given respondent from the average attitude of the respondents considered to represent the dominant culture. For each of the countries of origin we define

$$
\widetilde{\beta}_{(o, g, c)}=\beta_{(o, g, c)}-\beta_{(a v e, 4, c)}
$$

where $\widetilde{\beta}_{o, g, c}$ represents the difference of the country-origin effect, $\beta_{o, g, c}$, from the norm ( $\beta^{\prime} s$ here denote estimated values). To capture the multi-cultural nature of the U.S., we assume that the "norm" is represented by the weighted average of the attitudes of the fourth generation (or higher) of British, Irish, German, Italian, Polish and Scandinavian immigrants in our sample, $\beta_{\text {ave }, 4, c}$. We calculate the appropriate weights by using information from the 2000 U.S. Census about the ancestral composition of the non-foreign born population across different cohorts. ${ }^{23}$ In the robustness section we will experiment with different definitions of the norm.

To examine the experience of immigrants from different origins for the dynasty starting in the 1892-1916 period, we focus on two relationships. First, we compare $\widetilde{\beta}_{o, 1,1892-1916}$ to $\widetilde{\beta}_{o, 2,1917-1941}$, i.e. how the distance from the norm for members of the first generation of the dynasty compares to the distance from the norm for members of the second generation of the same dynasty. This relationship allows us to characterize the level of assimilation that occurs from the first to the second generation of immigrants of the same origin. We then compare $\widetilde{\beta}_{o, 1,1892-1916}$ to $\widetilde{\beta}_{o, 4, \geqslant 1967}$. We use this relationship to capture how the particular attitude of descendants changes from the first generation all the way to the fourth generation of the 1892-1916 dynasty, relative to the respective norms. ${ }^{24}$

This methodology follows and extends the approach proposed by in Algan, Bisin, Manning, and Verdier (2012). ${ }^{25}$ However, whereas they focus on the changes between the first and second generation, we analyze the evolution of attitudes over multiple generations. Most importantly, we keep the dynasty constant - only consider descendants of a "common original immigrant". This approach provides a rich, country of origin specific, picture of the process of cultural transmission, which is not contaminated by changes in attitudes of successive cohorts of immigrants.

It is useful to characterize the various patterns of convergence or non-convergence using a graph. Assume one plots the generation-1 deviation on the horizontal axis and the

\footnotetext{
${ }^{23}$ Although we can obtain the cohort specific frequency, we do not have the information to make it cohort and generation specific.

${ }^{24}$ One could also analyze the process of convergence between the first and third generation by comparing

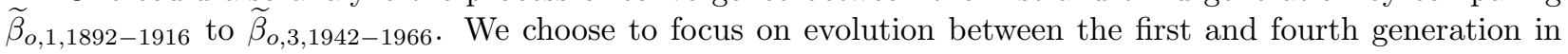
order to allow as much time as possible for attitudes to evolve further, beyond the change that occurs between the first and second generation.

${ }^{25} \mathrm{See}$, in particular, Figure 1.4 on p. 25.
} 
generation- 4 deviation on the vertical axis (i.e. $\widetilde{\beta}_{o, 1,1892-1916}$ and $\widetilde{\beta}_{o, 4, \geqslant 1967}$ ). We can partition the four quadrants in regions by drawing a 45 degree line and a 135 degree line going through the origin (see Figure 2a). Focusing on Quadrant I, with positive initial and final deviations from the norm, points between the x-axis and the 45 degree line represent monotonic convergence from above, in the sense that the deviation is larger in generation 1 than in generation 4, while those between the line and the y-axis capture monotonic divergence from above. Points between the (continuation of the) 45 degree line and the $\mathrm{x}$-axis in Quadrant III represent monotonic converge from below, while points between the 45 degree line and the y-axis monotonic divergence form below. In Quadrant II, in which the difference relative to the norm is first negative then positive, the 135 degree line separates points of divergent leapfrogging (above it) from those representing convergent leapfrogging (below the line). Similarly, in Quadrant IV, where the difference from the norm is first positive and then negative, points below the (continuation of the) 135 degree line are points of divergent regression and those above the line are points of convergent regression. This graph is useful to understand how the pattern of convergence differs for each cultural trait and each country.

We construct an overall index of convergence for each attitude by counting the proportion of countries that fall in the monotonic convergence from above or below, and in the convergent regression and convergent leapfrogging regions. In other terms we are counting, in this case, the points outside the hourglass defined by the 45 and 135 degree lines through the origin that represent a decrease in the absolute value of the distance from the norm going from the $1^{\text {st }}$ to the $4^{\text {th }}$ generation. Alternatively, we can do this for the $1^{\text {st }}$ and the $2^{\text {nd }}$ generation. We define the proportion of countries within these convergent region as $\pi_{45}$.

The drawback of $\pi_{45}$ is that it may not be a strict enough criterion. In particular it does not allow us to distinguish between slow-converging attitudes that feature country-generation effects close to the 45 degree line (or its reflection), and fast-converging ones clustered closer to the origin, along the y-axis. To this end, we define $\pi_{22.5}$ as the proportion of countries situated between the $\mathrm{x}$-axis and the 22.5 degree line (or its reflection). In other terms, we are now squeezing the hour-glass from above and count as convergent only those country-wave observations for which the absolute value of the distance from the norm in generation 1 has been cut at least in half by generation 4 (see Figure 2b). This is our preferred measure of convergence. One could use a somewhat tighter or looser criterion. As a robustness exercise, we will document in Section 7 that the ranking of attitudes obtained using the $\pi_{22.5}$ criterion is very similar to the one obtained when we require that the absolute value of the distance from the norm for generation 1 is cut by a third $\left(\pi_{30}\right)$ or two thirds by generation $4\left(\pi_{15}\right)$.

Note that this approach, particularly when using the $\pi_{45}$ criterion, is related to $\beta$ convergence as the latter focuses on whether the slope of the regression line of $\widetilde{\beta}_{o, 4, \geqslant 1967}$ on 
$\widetilde{\beta}_{o, 1,1892-1916}$ is between zero and one (so that the regression line lies in the monotonic convergence region). Yet, it is less parametric, less exposed to the influence of outliers, and it allows for convergent leapfrogging and convergent regression as well.

\section{Results}

In this section we present our results, using the definition of convergence based on the hourglass. We start by calculating the percentage of countries whose distance from the norm in generation 4 is less than half of the distance for their ancestor in generation 1. We use this fraction to quantify the convergence that occurs in a particular attitude (or group of attitudes) between the first and the fourth generation of the dynasty. As explained in the previous section, in the basic set of results we define the "norm" as the weighted average of the attitudes of the fourth (or higher) generation European immigrants in our sample. We compare the convergence that occurs by the fourth generation with that occurring between the first and the second generation.

After presenting the basic results we will explore in the next section several robustness exercises and extensions, such as tightening or relaxing the convergence criterion, using a reduced set of controls in the Probit equation, and changing the definition of the norm. We shall also present evidence on the changing strength of the relationship between attitudes in the country of origin and immigrants' attitudes across multiple generations. This issue is different, although is related, to the question whether or not attitudes converge to the norm. Moreover, it is an issue worth addressing because it has been studied by a number of authors in a context similar to ours, although with a focus limited to the second generation. ${ }^{26}$

The main results are summarized in Table 4 for groups of attitudes, and in Table 5 for individual attitudes and countries. In Table 4 we sort attitudes by the main groups shown in Table 1: Cooperation, Family, Gender, Religion, etc.. We denote with Gen $4 \pi_{22.5}$ the average across the attitudes in a given group of the fraction of convergent cases for each attitude. For example, $81 \%$ for "Cooperation" means that by the fourth generation $\left(G e n 4 \pi_{22.5}\right)$ the initial gap for the three attitudes related to cooperation (trust, fair and helpful) on average has been cut at least in half in $81 \%$ of all countries of origin. In the next column we show the same statistics for convergence by the second generation $\left(G e n 2 \pi_{22.5}\right)$, and in the third column the difference between the two, denoted by $\Delta$.

A number of common patterns emerge. First, whether a cultural trait can be considered persistent or not crucially depends upon whether one considers the change between the $1^{\text {st }}$ and $2^{\text {nd }}$ or the $1^{\text {st }}$ and $4^{\text {th }}$ generation. This point is very important: focusing only on the $2^{\text {nd }}$

\footnotetext{
${ }^{26}$ See the discussion and references in the Introduction.
} 
generation, as the literature has done so far, would miss the greater convergence of a number of attitudes.

Attitudes toward cooperation are slow moving initially, but eventually converge. By generation 2 the initial gap in these attitudes has been cut at least in half in only $33 \%$ of all countries of origin. By generation 4 this number has risen to $81 \%$, the largest percentage of convergent cases across all groups of attitudes. This suggests that while there is much to be gained in economic and social interaction from sharing attitudes towards cooperation - which is the reason why these attitudes eventually converge - it takes a long time for immigrants to realize this and change their attitudes. But convergence eventually occurs in most cases. Limiting the analysis to what happens between the 1 st and the 2 nd generation would lead to incorrect conclusions on the evolution of attitudes towards cooperation. Note that the $95 \%$ bootstrapped confidence interval for $\Delta$ does not include zero: we can thus reject the hypothesis of no change between the second and fourth generation in the attitudes toward cooperation.

A similar pattern emerges on average for attitudes toward the family (although, as we shall see, there are differences across individual attitudes): by generation 4 the percentage of convergent cases for the family category is $67 \%$, while it was $43 \%$ by generation 2 . As for attitudes toward cooperation, the $95 \%$ confidence interval for $\Delta$ does not include zero. For gender roles too there is a sizeable change in the percentage of convergent cases $(64 \%$ versus $43 \%$ ), but the $95 \%$ confidence interval now includes zero. Other groups of attitudes, while changing somewhat in the first two generations - which is natural following the shock of being exposed to a new society - remain quite different across country of origin and do not move much after the 2nd generation. By generation 4 the convergence percentage is $57 \%$ for abortion and the role of luck versus effort in determining social mobility, $43 \%$ for sexual behavior and views about the role of government, each essentially unchanged between Gen2 and Gen4. It is interesting that the groups that converge more slowly by the fourth generation are those connected with general political views, moral values concerning sexuality and abortion, and religion, while the fastest changing group of attitudes is the one related to cooperation, followed, perhaps surprisingly, by attitudes about the family (we shall discuss this result later in this section) and gender roles.

Sorting attitudes in our eight groups, as we have done in Table 4, helps get an overall picture of the way various types of attitudes evolve (if at all). Yet, there are (varying) degrees of heterogeneity within each group and the pace of convergence of individual attitudes, while more noisy, in some cases helps to better understand the mechanisms that may result in attitudes converging fast or slowly. The convergence of individual attitudes by generation 4 is shown in Table 5, which also contains information on convergence by country. The 
attitudes that converge less (with convergence proportions of less than $57 \%$ ) by the fourth generation are those that describe political views: helppoor (government should improve the standard of living of the poor), and polviews, but also eqwlth (should income be equalized between rich and poor) is rather slow moving. Attitudes towards sexual morality (premarsx, homosex), as well as abortion without restrictions (abany), are also among the slowest to converge. Interestingly, when one qualifies the access to abortion (abrisk: abortion restricted to cases of risk for the mother's health, defects in the fetus, or rape) there appears to be faster convergence. Most of the attitudes towards religion are in the next slower group (with convergence proportions of $57 \%$ ). There is instead heterogeneity among various family attitudes, with approval of sharing home with grown-up children (aged), frequency of evenings spent with relatives (socrel) and valuing children's independence (thnkself) converging more slowly, while attitudes towards divorce (divlaw) being the single fastest moving attitude. ${ }^{27}$ The slow convergence of getahead (work, help or luck as a source of social mobility) mirrors the slow convergence of general political attitudes and attitudes towards redistribution.

Among the attitudes that show the highest degree of convergence by generation 4 one finds all three attitudes about cooperation (helpful, trust, and fair). Interestingly, trust and fair are two of the slow moving attitudes when one focuses on the change between the first and second generation: for both attitudes by generation 2 the initial gap has been cut at least in half in only $14 \%$ of all countries of origin, while this percentage rises to $71 \%$ by generation 4 (it goes from $57 \%$ to $100 \%$ for the third attitude towards cooperation, helpful). The group of converging attitudes also contains one attitude towards religion (postlife), which is somewhat puzzling. Interestingly, the attitudes towards gender roles are split between those that are related to the labor market versus those that capture the role of women in politics: fechild (whether a child suffers when the mother works) is fast converging, while fepol (suitability of women for politics) is slow converging.

The behavior of individual attitudes, as mentioned above, is somewhat noisy: of the 26 attitudes we study a handful are hard to rationalize. Beyond the case of postlife, mentioned above, why do thnkself and obey converge at different speeds?. Beyond this handful of cases, however, the overall picture that emerges from analyzing both the 8 groups and the 24 individual attitudes, seems consistent with the predictions of our model of cultural transmission. Lazear (1999) and Konya (2005), from which we borrow our model of child identity choice, emphasize that cultural assimilation is more likely the greater the gain from sharing a cul-

\footnotetext{
${ }^{27}$ Inglehart and Baker (2000), using the World Value Survey (WVS), suggest that economic development is associated with shifts away from absolute norms and values toward more rational, tolerant, trusting, and participatory ones. However, they argue that cultural change is path dependent and is affected by the broad religious and cultural heritage of a society. Notice that many of the values and attitudes that we identify as slow moving are considered by Inglehart and Baker (2000) as characteristics that distinguish preindustrial from industrial societies.
} 
tural trait with the majority, and the greater the inefficiency of not doing so. Cavalli-Sforza (2001) also suggests that a trait is more likely to spread horizontally if it is beneficial (see also Tabellini 2008b). Our simple model indeed captures and further clarifies this effect, allowing for a parents' socialization choice, as in Bisin and Verdier (2001). In our model both the range of initial size of the minority for which full assimilation is the steady state equilibrium, and the speed of assimilation in each period, increase with the net transaction gain. This mechanism seems to be at work with many of the attitudes in our sample that appear to converge. For instance, there is much to be gained in economic and social interactions from sharing attitudes towards cooperation. One, however, has to wait the fourth generation for such attitudes to display a high level of convergence, a result which suggests that although there could be an initial gain from taking advantage of the trust of others, such gain is likely to be short-lived, followed by punishment if one is discovered cheating and not conforming to the social norm. ${ }^{28}$.

Within the gender group, the convergence of views regarding the cost of women working in terms of the quality of the relationship with their children can be explained by the large economic gains from having women participate in market work and the fact that generations of women have gradually learned about it. ${ }^{29}$ Conversely, it is interesting that attitudes that have to do with women's role in politics (fepol) display low convergence by generation 4 . We should not be surprised by the mixed results on gender norms, since many complex forces act on them. Previous empirical results are also mixed. Some authors (for instance, Goldin 2006 and Albanesi and Olivetti 2016) emphasize that technological innovations, structural change accompanying economic development, and medical improvements have had a powerful effect on gender roles in the labor market; instead, Alesina, Giuliano and Nunn (2013) find a persistent impact on gender norms today of the use of the plough as far back as a few millennia, even after accounting for the other factors mentioned above.

It also makes sense, in the light of our model, that general political orientation, attitudes towards redistribution and the role of effort versus luck in achieving success (also a component of one's overall ideological view) converge more slowly as they do not confer direct transactional advantages. Moreover, in a pluralistic and democratic society like the US, differences in political and ideological views are perfectly legitimate and can persist over time.

\footnotetext{
${ }^{28}$ The idea of attitudes towards cooperation as an important ingredient and lubricant of economic activity is a very old one and has received great attention recently. See, for instance, Fehr (2009) and the references therein on theoretical, econometric and experimental evidence on the consequences and determinants of trust. There is also an extensive literature on the role of schools in shaping attitudes towards cooperation. See, for instance, Algan, Cahuc and Shleifer (2013). Note that in deriving the country-generation effects we control for education of the respondent and of his/her parents.

${ }^{29}$ In our model, we do not allow for learning, See, however, Fernandez (2013) for a model of beliefs formation in which it takes time for people to update their beliefs about the implications for children's welfare of women working outside the home.
} 
The empirical evidence on the evolution/persistence of attitudes towards redistribution is mixed: Alesina and Fuchs-Schuendeln (2007), focus on German reunification and find that preferences concerning redistribution differ between East and West and that East Germans' preferences converge towards those of West Germans after unification; Luttmer and Singhal (2011), instead, suggest that preferences towards redistribution of immigrants still bear the hallmark of the country of origin. Our evidence is consistent with the results in the later paper in the sense that attitudes towards government redistributive intervention (particularly as summarized by helpoor) and general political beliefs display slow convergence. The process of ideology formation and the mechanisms through which views concerning the role of government in redistributing income can persist over time and can differ across countries have been studied in a related vast literature (see, for instance, Piketty (1995), Alesina and Glazer (2004), Alesina and Angeletos (2005), Benabou and Tirole (2006), Benabou (2008), and, for reviews, Alesina and Giuliano (2011) and Benabou and Tirole (2016)).

It is also understandable that attitudes towards sexuality, abortion, religion and some family attitudes should display slow convergence as it is plausible that these attitudes, may imply large gains for the parents if the child maintains the minority trait and, conversely, a large cost for the child if she abandons her family's traditional values and beliefs. Again, transactional gains are likely to be less important.

Our model is suggestive of the reasons why private attitudes towards religion or attitudes that do not imply an outward manifestation of one's convictions (such as pray and prayer) should display slow convergence. But the same argument does not apply to attitudes that are embodied or likely to be embodied in public, rather than private, manifestations of one's beliefs (such as attend): the reason could be that conforming (for instance attending a service on Sunday independently of one's religion) may confer social benefits. Gruber and Hungerman (2008) indeed find that changes in shopping hours had a large impact on church attendance and conclude that this validates economic models of religiosity that highlight the importance of economic influences, such as the opportunity cost of church-going for religious participation.

An interesting question is whether the probability that a cultural attitude converges or not depends upon how spread out across countries of origin is the distribution of the trait in the first generation. Here the arguments go both ways: large differences in the first generation may make a movement towards the norm more advantageous; on the other hand, reducing distances may be more difficult if distances are more spread out. In our results the median initial standard deviation of the seven faster moving attitudes is only slightly larger than the median standard deviation for the slower moving ones (.41 versus .39). Thus the initial dispersion of opinions among first generation immigrants regarding each attitude does not seem to play a role in the ensuing convergence in subsequent generations. 
Our results have implications for the debate between the views that emphasize the assimilation of immigrants, versus those that highlight the preservation of a separate identity, and for the question whether the melting pot metaphor is an accurate description of immigrants' experience in the US. We find that by the fourth generation, for all countries but one (Poland), the majority of cultural attitudes of descendants of European and Mexican immigrants has converged, consistently with Assimilation Theory (see the bottom row of Table 5 that reports the total fraction of convergent attitudes by the fourth generation for each country). However, contrary to the prediction of that theory, and consistently with Multiculturalism, descendants of immigrants from different countries of ancestry have maintained over several generations a degree of cultural distinctiveness along some traits. In other words, the temperature in the melting pot is hot, but not uniform throughout, as one would expect given the model of cultural transmission we have developed that points to the fact that the speed of convergence is likely to differ across attitudes.

Are there interesting country specificities in the pattern of convergence? In addition to Great Britain, the country with the largest weight in defining the norm, Ireland and Germany show the highest number of converging attitudes. Poland is at the bottom with Mexico, Italy and Scandinavia. Which factors could explain the number of convergent attitudes by country? One would expect, on average, that in countries of origin in which the family is a weaker social institution, direct transmission of traits would be relatively less important or effective. This is captured in our model by the parameters representing the benefit to the parent from the child maintaining the original trait, and by the effectiveness of the socialization technology embodied in the portion of the child's switching cost related to the parent's educational efforts. To investigate the role of the family we use a measure of the strength of family ties proposed by Alesina and Giuliano (2010). This measure captures beliefs on the importance of the family in a person's life, the duties and responsibilities of parents and children, and the love and respect for one's own parents. The data come from answers to a set of World Value Service questions. ${ }^{30}$ This measure yields the following ranking for our countries (from the weakest to the strongest ties): Germany, Scandinavia, UK, Ireland, Poland, Italy, Mexico. With the exception of Scandinavia, this ranking of family ties is very similar to the ranking for the proportion of convergent attitudes: indeed the rank correlation coefficient is positive $(r=.62)$. This suggests that family strength plays an important role in reducing the speed of convergence of immigrants' attitudes to the prevailing norm. However, it is not the only factor. The ease to learn English may also matter for convergence to the norm. In our model this is captured by the distribution of the stochastic component of the child switching costs. As a proxy for the ability to acquire English proficiency we use the average, for each country of

\footnotetext{
${ }^{30}$ See Section 2.1.2 for details and Figure 1 for the ranking of countries.
} 
origin, of the number of words (out of ten) of which $1^{\text {st }}$ generation immigrants can identify the meaning ${ }^{31}$. The rank correlation with the number of convergent attitudes for the countries in our sample is positive and equal to .43. Finally, the number of convergent attitudes by country is negatively correlated $(r=-.56)$ with the measure of residential segregation used in Borjas 1995. ${ }^{32}$ Although our model is silent on this issue, as it does not include a residential choice, this is what one would expect since a neighborhood characterized by a high concentration of individuals from the same ancestry is likely to contribute to perpetuating the culture of the country of origin and to a slowing down of the process of cultural integration. The issue of how the composition of the neighborhood affects the evolution of individuals' attitudes is a very important and interesting topic that we leave for future research.

\section{$7 \quad$ Robustness and Extensions}

In this section we discuss several robustness exercises. Are our results robust, for instance, to a change in the tightness of the convergence criteria in terms of the definition of the convergence region? Are they robust to the menu of controls included in the Probit model used to measure the country-generation-cohort effects or to changes in the definition of the norm to which attitudes converge? The answer to these questions, as we shall see in the next three sub-sections, is mostly yes.

Finally, in the last sub-section we extend our analysis to a related, but quite distinct issue: how do the cultural attitudes of succeeding generations of immigrants relate to those of individuals who have not migrated and kept living in the country of origin? In particular, do we observe a weakening of the relationship as the temporal distance from the country of origin increases over generations? We will also ask how the attitudes of various generations of immigrants are related to those prevailing in the country of origin for the cohort from which the first generation of immigrants was drawn. Although the issue of distance from the contemporary or ancestral culture in the country of origin and the main question addressed in this paper - convergence to the prevailing norm in the country of immigration - are different, we address it since it has been studied by a number of authors in a context similar to ours.

\footnotetext{
${ }^{31}$ The GSS includes a series of questions that identify the respondent's vocabulary ability.

${ }^{32}$ More precisely, see Borjas (1995), Table 2. We use the measure based on the percentage of first and second generation immigrants in the neighborhood of the same ethnicity as a first-generation immigrants. Similar results are obtained using figures for the second generation.
} 


\subsection{Changing the Definition of the Convergence Region}

In our baseline results we have measured convergence focusing, for each attitude, on the index we called $\pi_{22.5}$, which measures the proportion of countries that have cut the absolute value of the distance of generation 4 from the norm by at least half relative to generation 1 . In Table A1 of Appendix 1 we present detailed results for the $4^{\text {th }}$ generation based on less or more stringent criteria for convergence: reducing that distance by any amount $\left(\pi_{45}\right)$, by at least a third $\left(\pi_{30}\right)$, and by at least two thirds $\left(\pi_{15}\right)$. The (Spearman) rank correlation coefficients between the proportions of converging country-wave observations (by generation 4) in the baseline and those obtained using these alternative criteria are reported at the bottom of the table. Using $\pi_{30}$ or $\pi_{15}$, instead of $\pi_{22.5}$, leaves the ranking of the degree of convergence of the different groups of attitudes by and large unchanged. Correlation coefficients with the ranking in our baseline case for individual attitudes are also very high (in excess of $72 \%$ ). Moreover, the conclusion that it is important to go beyond the second generation in assessing convergence also still holds. The correlation with the ranking obtained when using $\pi_{45}$ is instead smaller (.54) and the difference in convergence speed across groups less sharp (although cooperation remains the fastest convergent group). This is not surprising and, as we have already argued, we find convergence by any amount the least convincing criterion.

\subsection{Reducing the Set of Controls in the Probit Equation}

In Table A2 we limit the set of common controls only including age, age squared, year-of-thesurvey dummy, gender, religion, regional indicators, and urbanization indicators. Income, education, mother's education, father's education, number of children, marital status, and work status are excluded: in the case of income and education level of a respondent because they are very likely to influence some of his/her attitudes. In turn, both income and education (as well as the rest of the excluded individual controls) can be viewed as an outcome of factors encoded in the country of origin fixed effects. The main results in the paper focus on the ancestral influence on attitudes that are not explained by variations in income, education, etc.. In Table A2, instead, one allows for the ancestral influence to affect attitudes both directly and through changes in these endogenous individual characteristics. While the convergence rates by generation four of attitudes about cooperation, political views, religion, family and reasons for success are similar, now attitudes towards gender, sexuality and abortion display a higher convergence proportion. The Spearman rank correlation coefficients with our original ranking for individual attitudes is rather low and equal to 0.25 , which emphasizes the importance of controlling for income, education, etc.. For the cooperation, gender, family and sexual behavior groups, the change between generation 2 and generation 4 convergence is now 
larger, which is what one would expect since now the change captures also the convergence in education levels across groups.

\subsection{Changing the definition of the Norm}

In Table A3 we return to our baseline specification and experiment changing the definition of the norm. More specifically, instead of defining the norm as the weighted average of the attitudes of the fourth (or higher) generation European immigrants in our sample, we choose as reference point the fourth generation descendants of immigrants from Great Britain. The rank correlation coefficient with our original ranking is .57 and our conclusions remain largely the same. This should not be surprising since descendants of British immigrants represent a large share (around 40\%) of the immigrants who are fourth generation (or higher). Our conclusions are also unchanged when we include Mexico in the calculation of the norm, together with the other European countries (see Table A4). The correlation coefficient with the ordering in the basic specification is now 0.88 .

\subsection{Immigrants' Attitudes and Attitudes in the Country of Origin}

When assessing the strength of the association between immigrants' culture and the culture of the country of ancestry - which, as we explained, is a question different but related to the one addressed in this paper - there are two possible ways to proceed. We could focus on the relationship of immigrants' attitudes with those of the corresponding cohort in the country of origin. Alternatively we could compare immigrants' attitudes with attitudes in the country of origin for the cohort to which the first generation of immigrants belonged and from which the various generations descend. In the former case the reference point is the "contemporary" (same cohort) culture. In the alternative it is the "ancestral" culture of the country of origin, that is the culture the founder of the dynasty brought with him/her when he/she first migrated to the US. We shall conduct both exercises for different generations of immigrants.

We measure attitudes in the countries of origin using the European Value Survey (EVS) and the World Value Survey (WVS) which ask very similar questions, some of which coincide - often are almost identical - to those asked in the GSS and used in our baseline results. The match between the two surveys is very close for the questions regarding some of the cultural attitudes we have used in our empirical work, such as trust, attend, postlife, and homosex, and a fairly close (but not perfect) for pray, thnkself, obey, fechild, fework, and abany (See Table A5). The match is not close for the remaining attitudes we have examined. We have pooled the EVS and WVS data for all the relevant countries for the periods matching those defined 
in our baseline model. In the first stage, we have estimated the coefficient of country-cohort specific dummies in a Probit model for each attitude, controlling for survey-year effects, age, age squared, gender, and marital status. ${ }^{33}$ In the second stage, we have then associated these country-cohort-specific effects with the data in each GSS survey, so that each individual as been matched with the culture in the country of origin of the cohort she/he belongs to. We have then estimated the Probit models for each cultural attitude on the GSS data, as we did before, but replacing the period-origin-generation and origin-cohort dummies with the time-varying and country-specific cultural proxies obtained in the first stage, interacted with generation dummies. We continue to control for all the individual specific variables used before and for common year effects. Essentially, we are assuming that the country of origin and cohort specific movements in culture for US immigrants are proportional to the cultural proxy estimated in the first stage, and that its effect may vary across generations. In particular, we are interested in assessing the significance of the generation-specific coefficients and whether the effect of the culture of origin decreases (or not) going from the $1^{\text {st }}$ to the $4^{\text {th }}$ generation. We have then repeated this exercise by matching to each respondent the country of origin specific effects of the cohort of first emigration immigrants, to capture the ancestral culture of the founder of each dynasty of immigrants.

The results for both experiments are reported in Table 6, Part 1 (same cohort), and Part 2 (cohort of dynasty founder). First, considering all attitudes, in eight out of ten cases the coefficients of the culture of the country of origin for the first generation are significant at conventional levels or nearly so, whatever the reference point of the country of origin. The association is closest for the attitudes that bear a close correspondence in the actual question surveyed in the GSS and in the EVS-WVS. This emphasizes the fact that an imperfect match between the EVS-WVS and the GSS questions is likely to lead to underestimating the strength of the association with the culture of the country of origin. In four cases the association is significant for the second generation at the $5 \%$ level and for one case at the $10 \%$ level (or nearly so), both in Part 1 and in Part 2. Although, in the case of trust, attend, pray, fechild, and homosex, there are differences in the significance level, depending upon the reference point. Most interestingly, from our point of view, the value of the generation specific coefficients decreases in most cases as we go from the first to higher generations, implying a weakening of the effect of the culture of the country of origin, whether contemporary or ancestral. For instance, in the case of trust the coefficients for all generations are always significant, but decrease (when the reference point is the corresponding generation in the country of origin) from .39 to $.34, .27, .23$ as we go from the $1^{\text {st }}$ to the $4^{\text {th }}$ generation. This means that, when assessing the strength of association with the culture of the country of

\footnotetext{
${ }^{33}$ The results that follow are not sensitive to the choice of the controls.
} 
origin across generations, it is important to go beyond the second generation to have a full picture, as it was the case when analyzing convergence.

When we compare attitudes of the same cohort (one living in the US, the other in the country of origin), a weakening of the association from the first to higher generations does not imply nor is implied by convergence to the norm in the US. For instance, differences in attitudes across immigrants of distinct ethnicity (the issue investigated in this paper) could persist, and still the attitudes of immigrants could drift away from those prevailing in the country from which their ancestors originally came. Alternatively, one's cultural traits may remain close to those of the country of origin, but convergence to the norm across generations may be observed because over time values across countries become more similar. Analogous considerations apply when we use, as reference point, the culture that the founder of the dynasty brought with him/her when he/she first migrated to the US. In practice, a weakening of the association with the founder's ancestral culture is likely to be a precondition for convergence to the norm for successive generations of immigrants within a dynasty.

\section{Conclusions}

Are immigrants' values and beliefs deeply rooted in the culture of the country of origin, so that they persist relatively unchanged across generations, or do change in response to the new economic and social environment and converge rather rapidly to the prevailing norm of the recipient country? Answering this question is an important step in addressing the more general problem of how persistent a society's values and beliefs are - an issue on which there is abundant disagreement In this paper we have presented new evidence on this question by analyzing cultural attitudes of different generations of European and Mexican immigrants to the US, and we have provided a simple model to shed light and interpret the evidence on the speed of convergence.

We find that persistence is not the same across cultural traits. Some show a higher degree of convergence to the prevailing norm: this is true, for example, for attitudes towards cooperation (the trustworthiness, helpfulness and fairness of others), and towards the effect of women's work on the child-mother relationship, and some family attitudes, such as views on divorce. Other traits, instead, show a lower degree of convergence: for instance attitudes towards politics and redistribution, sexuality, abortion, religious values, and some family attitudes such as sharing home with grown-up children and frequency of evenings spent with relatives. A higher degree of convergence appears to characterize attitudes for which the benefits of assimilation are likely to be greater; instead, attitudes that are either characterized by lower benefits or for which direct transmission within the family is likely to be more 
important and effective show slower convergence.

Importantly we also find that one would not come to these conclusions if one limited the analysis to just the first two generations of immigrants, as the literature has so far mostly done. Focusing only on the first two generations biases the conclusion in favor of persistence. Finally, we show that persistence is "culture specific" in the sense that the country from which one's ancestors came matters for the pattern of generational convergence (or lack thereof). The strength of family ties, the ability to learn English and residential segregation appear to be important factors in this respect.

The implication of our results for the debate about the "melting pot" is that for manycultural traits and beliefs a melting-pot effect was certainly at work among immigrants. For other traits, however, descendants of immigrants from different countries of ancestry have maintained over several generations a degree of cultural distinctiveness. Thus, the temperature in the melting pot was hot, but not uniform throughout, as suggested by our model that emphasizes how the effectiveness and importance of the socialization mechanism by parents and of the benefits from assimilation for their children is likely to vary across attitudes and countries.

Finally, one may ask whether the evidence provided in this paper has any relevance for the question concerning the likelihood of success of reforms designed to change practices within a country. Are such reforms doomed because a country's culture cannot be changed, or can they succeed because they can change cultural attitudes by altering incentives? This paper neither intends to, nor can provide an answer to this question. What we have shown, however, is that the large shock represented by the new social and economic environment faced by immigrants can eventually lead to a change in many cultural traits. We have also found that the process of change depends upon cultural characteristics of the country of origin, so that any answer is likely to be country specific. These issues are fertile ground for future research. 


\section{$9 \quad$ Tables and Figures}

Table 1: List of Attitudes: Groups, Abbreviations, Descriptions

\begin{tabular}{|c|c|c|}
\hline Group A - Cooperation & \begin{tabular}{|l|l|} 
trust \\
fair \\
helpful
\end{tabular} & $\begin{array}{l}\text { can people be trusted or cannot be too careful? }\left(\mathrm{y}=1 \text { for yes if } x_{G S S}=1\right) \\
\left.\text { will people take advantage of you? ( } \mathrm{y}=1 \text { for no if } x_{G S S}=2\right) \\
\text { people are mostly helpful or looking out for themselves }\left(\mathrm{y}=1 \text { for yes if } x_{G S S}=1 \text { ) }\right.\end{array}$ \\
\hline Group B - Government/Politics & \begin{tabular}{|l|l|} 
eqwlth \\
helppoor \\
polviews
\end{tabular} & $\begin{array}{l}\text { government should equalize income between poor and rich }\left(\mathrm{y}=1 \text { for yes if } x_{G S S}<5\right) \\
\text { government should improve the standard of living of the poor }\left(\mathrm{y}=1 \text { for yes if } x_{G S S}<4\right) \\
\text { political views }\left(\mathrm{y}=1 \text { for liberal if } x_{G S S}<4\right)\end{array}$ \\
\hline Group C - Religion & $\begin{array}{l}\text { attend } \\
\text { pray } \\
\text { reliten } \\
\text { postlife } \\
\text { prayer }\end{array}$ & $\begin{array}{l}\text { frequency of religious services attendance }\left(\mathrm{y}=1 \text { for less often if } x_{G S S}<5\right) \\
\text { frequency of prayer }\left(\mathrm{y}=1 \text { for less often if } x_{G S S}>4\right) \\
\text { intensity of religious affiliation }\left(\mathrm{y}=1 \text { for not strong if } x_{G S S}>1\right) \\
\text { belief in life after death }\left(\mathrm{y}=1 \text { for no if } x_{G S S}=2\right) \\
\text { approval of prayer in public schools }\left(\mathrm{y}=1 \text { for disapprove if } x_{G S S}=2\right)\end{array}$ \\
\hline Group D - Family & $\begin{array}{l}\text { thnkself } \\
\text { obey } \\
\text { pillok } \\
\text { aged } \\
\text { divlaw } \\
\text { socrel }\end{array}$ & $\begin{array}{l}\left.\text { independence of a child is highly important quality ( } \mathrm{y}=1 \text { for important if } x_{G S S}<3\right) \\
\left.\text { obedience of a child is a highly important quality ( } \mathrm{y}=1 \text { for not important if } x_{G S S}>2\right) \\
\text { birth control available to teenagers without parental consent }\left(\mathrm{y}=1 \text { for ok if } x_{G S S}<3 \text { ) }\right. \\
\text { approval of sharing home with grown children }\left(\mathrm{y}=1 \text { for disapproval if } x_{G S S}>1\right) \\
\left.\text { should divorce be easier? ( } \mathrm{y}=1 \text { for yes if } x_{G S S}=1,3\right) \\
\left.\text { frequency of social evenings with relatives ( } \mathrm{y}=1 \text { for less often if } x_{G S S}>3\right)\end{array}$ \\
\hline Group E - Gender Roles & $\begin{array}{l}\text { fechild } \\
\text { fepol }\end{array}$ & $\begin{array}{l}\text { working mother can have a good relationship with children }\left(\mathrm{y}=1 \text { for yes if } x_{G S S}<3\right) \\
\text { women not suited for politics }\left(\mathrm{y}=1 \text { for no if } x_{G S S}=2\right)\end{array}$ \\
\hline Group F - Abortion & $\begin{array}{l}\text { abany } \\
\text { abrisk }\end{array}$ & $\begin{array}{l}\text { approval of abortion for any reason }\left(\mathrm{y}=1 \text { for yes if } x_{G S S}=1\right) \\
\text { approval of abortion for health/defect/rape reasons }\left(\mathrm{y}=1 \text { for yes if } x_{G S S}=0\right)\end{array}$ \\
\hline Group G - Sexual Behavior & $\begin{array}{l}\text { premarsx } \\
\text { homosex }\end{array}$ & $\begin{array}{l}\text { approval of premarital sex }\left(\mathrm{y}=1 \text { for yes if } x_{G S S}=4\right) \\
\text { approval of same-sex sexual relations }\left(\mathrm{y}=1 \text { for yes if } x_{G S S}>2\right)\end{array}$ \\
\hline Group H - Mobility/Success & getahead & work, help, luck as a source of social mobility ( $\mathrm{y}=1$ for work if $x_{G S S}=1$ ) \\
\hline
\end{tabular}

Table 2: Countries and Country Groups

\begin{tabular}{l|l}
\multicolumn{2}{c}{ Country Group } \\
\hline British origin (GB) & England, Wales, Scotland \\
German origin (GER) & Germany \\
Irish origin (IRE) & Ireland \\
Italian origin (ITA) & Italy \\
Polish origin (POL) & Poland \\
Scandinavian origin (SCA) & Denmark, Finland, Sweden, Norway \\
Mexican origin (MEX) & Mexico \\
\hline
\end{tabular}


Table 3: Number of Respondents for the Question on Trust by Origin, Cohort, and Generation

\begin{tabular}{|c|c|c|c|c|c|c|c|c|c|c|c|c|c|c|c|c|}
\hline \multirow[t]{2}{*}{ Part 1: } & \multicolumn{4}{|c|}{ Cohort 1892-1916 } & \multicolumn{4}{|c|}{ Cohort 1917-1941 } & \multicolumn{4}{|c|}{ Cohort 1942-1966 } & \multicolumn{4}{|c|}{ Cohort 1967+ } \\
\hline & Gen1 & Gen2 & Gen3 & Gen 4 & Gen1 & Gen2 & Gen3 & Gen4 & Gen1 & Gen2 & Gen3 & Gen4 & Gen1 & Gen2 & Gen3 & Gen4 \\
\hline GER & 13 & 71 & 60 & 118 & 46 & 78 & 302 & 632 & 66 & 87 & 345 & 1,579 & 29 & 38 & 57 & 536 \\
\hline POL & 13 & 48 & 4 & 1 & 14 & 81 & 59 & 14 & 26 & 30 & 207 & 81 & 6 & 7 & 17 & 62 \\
\hline SCA & 12 & 57 & 15 & 4 & 10 & 72 & 124 & 71 & 16 & 28 & 183 & 307 & 6 & 3 & 17 & 112 \\
\hline IRE & 8 & 33 & 28 & 121 & 11 & 53 & 158 & 493 & 26 & 44 & 233 & 1,153 & 11 & 19 & 48 & 445 \\
\hline ITA & 20 & 54 & 3 & 1 & 37 & 180 & 74 & 13 & 37 & 86 & 387 & 173 & 7 & 28 & 71 & 186 \\
\hline GB & 21 & 43 & 49 & 237 & 59 & 82 & 123 & 1,017 & 69 & 83 & 166 & 1,501 & 21 & 17 & 25 & 420 \\
\hline MEX & 2 & 3 & 0 & 3 & 27 & 45 & 13 & 12 & 151 & 110 & 86 & 73 & 263 & 165 & 42 & 76 \\
\hline
\end{tabular}

Part 2: $\quad$ Dynasty 1892-1916

\begin{tabular}{lcccc} 
& Gen1 & Gen2 & Gen3 & Gen4 \\
\cline { 2 - 5 } GER & 59 & 78 & 345 & 536 \\
POL & 27 & 81 & 207 & 62 \\
SCA & 22 & 72 & 183 & 112 \\
IRE & 19 & 53 & 233 & 445 \\
ITA & 57 & 180 & 387 & 186 \\
GB & 80 & 82 & 166 & 420 \\
MEX & 29 & 45 & 86 & 76
\end{tabular}

Notes: In part 2 we assume that of the first generation of the 1892-1916 and 1917-1941 cohort share the same attitude towards trust.

Table 4: Convergence of Cultural Attitudes (by Groups): Comparing Generation 4 and 2

\begin{tabular}{|c|c|c|c|c|c|c|}
\hline & & Gen $4 \pi_{22.5}$ & Gen $2 \pi_{22.5}$ & $\triangle$ & $90 \% \mathrm{CI}$ & $95 \% \mathrm{CI}$ \\
\hline Group A - Cooperation & $\begin{array}{l}\text { trust } \\
\text { fair } \\
\text { helpful }\end{array}$ & $81 \%$ & $33 \%$ & $48 \%$ & $(14 \%, 48 \%)$ & $(10 \%, 52 \%)$ \\
\hline Group B - Government & $\begin{array}{l}\text { eqwlth } \\
\text { helppoor } \\
\text { polviews }\end{array}$ & $38 \%$ & $43 \%$ & $-5 \%$ & $(0 \%, 43 \%)$ & $(0 \%, 43 \%)$ \\
\hline Group C - Religion & $\begin{array}{l}\text { attend } \\
\text { pray } \\
\text { reliten } \\
\text { postlife } \\
\text { prayer }\end{array}$ & $60 \%$ & $46 \%$ & $14 \%$ & $(3 \%, 34 \%)$ & $(0 \%, 37 \%)$ \\
\hline Group D - Family & $\begin{array}{l}\text { thnkself } \\
\text { obey } \\
\text { pillok } \\
\text { aged } \\
\text { divlaw } \\
\text { socrel }\end{array}$ & $67 \%$ & $43 \%$ & $24 \%$ & $(10 \%, 38 \%)$ & $(7 \%, 40 \%)$ \\
\hline Group E - Gender Roles & $\begin{array}{l}\text { fechld } \\
\text { fepol }\end{array}$ & $64 \%$ & $43 \%$ & $21 \%$ & $(-7 \%, 43 \%)$ & $(-7 \%, 50 \%)$ \\
\hline Group F - Abortion & $\begin{array}{l}\text { abany } \\
\text { abrisk }\end{array}$ & $57 \%$ & $50 \%$ & $7 \%$ & $(-7 \%, 36 \%)$ & $(-7 \%, 43 \%)$ \\
\hline Group G - Sexual Behavior & $\begin{array}{l}\text { premarsx } \\
\text { homosex }\end{array}$ & $43 \%$ & $43 \%$ & $0 \%$ & $(0 \%, 43 \%)$ & $(-7 \%, 50 \%)$ \\
\hline Group H - Mobility/Success & getahead & $57 \%$ & $57 \%$ & $0 \%$ & $(-29 \%, 43 \%)$ & $(-29 \%, 57 \%)$ \\
\hline \multicolumn{7}{|c|}{$\begin{array}{l}\text { Notes: Convergence is achieved when the absolute value of the deviation from the norm has been } \\
\text { cut at least in half between generation } 1 \text { and generation } 4 \text { or } 2\left(\pi_{22.5} \text { criterion). Gen } 4 \pi_{22.5} \text { denotes }\right. \\
\text { the average percentage of convergent cases by generation } 4 \text { within each group, and Gen } 2 \pi_{22.5} \text { by } \\
\text { generation } 2 . \triangle \text { denotes the difference in the percentage of convergent cases between generations } 4 \\
\text { and generation } 2 . \text { The last two columns report the bootstrapped } 90 \% \text { and } 95 \% \text { confidence intervals } \\
\text { for } \triangle \text {, based on } 500 \text { replications estimating the Probit equation, based on stratified sampling with } \\
\text { replacement in the country-generation-cohort cells. }\end{array}$} \\
\hline
\end{tabular}


Table 5: Convergence by Each Cultural Attitude and Country

\begin{tabular}{llcccccccc}
\hline & & Gen $4 \pi_{22.5}$ & GER & POL & SCA & IRE & ITA & GB & MEX \\
\hline \multirow{3}{*}{ Group A - Cooperation } & trust & $71 \%$ & 1 & 1 & 1 & 1 & 0 & 1 & 0 \\
& fair & $71 \%$ & 1 & 0 & 1 & 1 & 1 & 1 & 0 \\
& helpful & $100 \%$ & 1 & 1 & 1 & 1 & 1 & 1 & 1 \\
\hline \multirow{3}{*}{ Group B - Government } & eqwlth & $57 \%$ & 1 & 0 & 1 & 1 & 1 & 0 & 0 \\
& helppoor & $29 \%$ & 1 & 0 & 0 & 0 & 0 & 1 & 0 \\
& polviews & $29 \%$ & 0 & 0 & 0 & 0 & 0 & 1 & 1 \\
\hline \multirow{3}{*}{ Group C - Religion } & attend & $57 \%$ & 1 & 0 & 0 & 1 & 1 & 0 & 1 \\
& pray & $57 \%$ & 1 & 0 & 0 & 1 & 0 & 1 & 1 \\
& reliten & $57 \%$ & 1 & 0 & 0 & 1 & 1 & 1 & 0 \\
& postlife & $71 \%$ & 1 & 0 & 1 & 1 & 1 & 0 & 1 \\
& prayer & $57 \%$ & 0 & 0 & 0 & 1 & 1 & 1 & 1 \\
\hline \multirow{3}{*}{ Group D - Family } & thnkself & $57 \%$ & 1 & 1 & 0 & 0 & 1 & 1 & 0 \\
& obey & $71 \%$ & 0 & 1 & 0 & 1 & 1 & 1 & 1 \\
& pillok & $71 \%$ & 1 & 0 & 1 & 1 & 0 & 1 & 1 \\
& aged & $43 \%$ & 0 & 0 & 0 & 1 & 1 & 0 & 1 \\
& divlaw & $100 \%$ & 1 & 1 & 1 & 1 & 1 & 1 & 1 \\
& socrel & $57 \%$ & 1 & 0 & 0 & 0 & 1 & 1 & 1 \\
\hline \multirow{2}{*}{ Group E - Gender Roles } & fechld & $71 \%$ & 1 & 1 & 1 & 0 & 1 & 0 & 1 \\
& fepol & $57 \%$ & 1 & 1 & 1 & 0 & 0 & 1 & 0 \\
\hline \multirow{2}{*}{ Group F - Abortion } & abany & $43 \%$ & 1 & 0 & 0 & 1 & 0 & 1 & 0 \\
& abrisk & $71 \%$ & 1 & 1 & 1 & 1 & 0 & 0 & 1 \\
\hline \multirow{2}{*}{ Group G - Sexual Behavior } & premarsx & $43 \%$ & 1 & 0 & 1 & 0 & 0 & 0 & 1 \\
& homosex & $43 \%$ & 0 & 1 & 1 & 1 & 0 & 0 & 0 \\
\hline Group H - Mobility/Success & getahead & $57 \%$ & 1 & 0 & 1 & 0 & 1 & 1 & 0 \\
\hline \multirow{2}{*}{ at } & & & $79 \%$ & $38 \%$ & $54 \%$ & $67 \%$ & $58 \%$ & $67 \%$ & $58 \%$ \\
\hline
\end{tabular}

Notes: The figures in the table represent the number of times we observe convergence for each country and each attitude ( 1 denotes convergence). Convergence is achieved when the absolute value of the deviation from the norm has been cut at least in half between generation 1 and generation $4\left(\pi_{22.5}\right.$ criterion). Gen $4 \pi_{22.5}$ denotes here the percentage of convergence cases for each attitude.

\section{Table 6: Relationship between Attitudes in the Country of Origin and Attitudes of US Im- migrants across Generations}

Part 1: Relationship with contemporary attitudes

\begin{tabular}{lccccccccc} 
Variable & trust & attend & pray & postlife & thnkself & obey & fechild & abany & homosex \\
\hline Contemporary Culture $_{o} \times I_{(g=1)}$ & 0.39 & 0.31 & 0.39 & 0.77 & 0.42 & 0.36 & 0.18 & 0.00 & 0.09 \\
& $(5.12)$ & $(4.47)$ & $(4.91)$ & $(3.55)$ & $(3.83)$ & $(2.04)$ & $(3.76)$ & $(-0.08)$ & $(1.81)$ \\
ContemporaryCulture $_{o} \times I_{(g=2)}$ & 0.34 & 0.10 & 0.17 & 0.08 & 0.11 & 0.08 & 0.10 & -0.04 & 0.11 \\
& $(4.96)$ & $(1.94)$ & $(2.49)$ & $(0.65)$ & $(1.36)$ & $(0.59)$ & $(2.61)$ & $(-0.96)$ & $(2.41)$ \\
ContemporaryCulture $_{o} \times I_{(g=3)}$ & 0.26 & 0.10 & 0.05 & 0.22 & 0.02 & 0.08 & 0.08 & 0.01 & 0.03 \\
& $(4.25)$ & $(3.39)$ & $(0.97)$ & $(2.91)$ & $(0.24)$ & $(1.05)$ & $(2.22)$ & $(0.39)$ & $(0.79)$ \\
Contemporary Culture $_{o} \times I_{(g=4)}$ & 0.22 & -0.02 & 0.04 & -0.05 & -0.02 & -0.14 & 0.10 & 0.03 & 0.09 \\
& $(3.59)$ & $(-1.00)$ & $(1.16)$ & $(-1.08)$ & $(-0.28)$ & $(-2.90)$ & $(2.63)$ & $(0.86)$ & $(2.26)$ \\
\hline
\end{tabular}

Part 2: Relationship with ancestral attitudes

\begin{tabular}{lccccccccc} 
Variable & trust & attend & pray & postlife & thnkself & obey & fechild & abany & homosex \\
\hline AncestralCulture $_{o} \times I_{(g=1)}$ & 0.52 & 0.35 & 0.41 & 0.66 & 0.56 & 0.38 & 0.21 & 0.08 & 0.14 \\
& $(4.72)$ & $(4.91)$ & $(4.93)$ & $(3.07)$ & $(3.65)$ & $(2.06)$ & $(2.52)$ & $(1.17)$ & $(1.58)$ \\
AncestralCulture $_{o} \times I_{(g=2)}$ & 0.49 & 0.20 & 0.21 & 0.08 & 0.13 & 0.09 & 0.08 & 0.05 & 0.11 \\
& $(4.90)$ & $(3.34)$ & $(2.53)$ & $(0.63)$ & $(1.22)$ & $(0.51)$ & $(1.42)$ & $(0.85)$ & $(1.52)$ \\
AncestralCulture $_{o} \times I_{(g=3)}$ & 0.32 & 0.09 & 0.04 & 0.10 & -0.01 & 0.27 & 0.04 & 0.05 & 0.02 \\
& $(3.71)$ & $(2.16)$ & $(0.64)$ & $(1.11)$ & $(-0.18)$ & $(1.60)$ & $(0.95)$ & $(0.93)$ & $(0.45)$ \\
AncestralCulture $_{o} \times I_{(g=4)}$ & 0.31 & -0.12 & 0.02 & -0.09 & 0.02 & -0.07 & 0.02 & 0.09 & 0.10 \\
& $(3.22)$ & $(-2.89)$ & $(0.37)$ & $(-1.10)$ & $(0.36)$ & $(-0.41)$ & $(0.62)$ & $(1.62)$ & $(2.04)$ \\
\hline
\end{tabular}

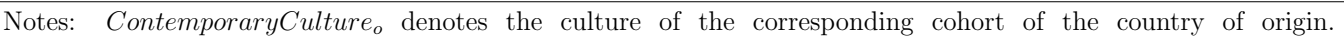
AncestralCulture $e_{o}$ denotes the culture of the cohort from the country of origin which originates the dynasty which the immigrant belongs to. Generation specific coefficients are reported. $z$ statistics in parentheses. 


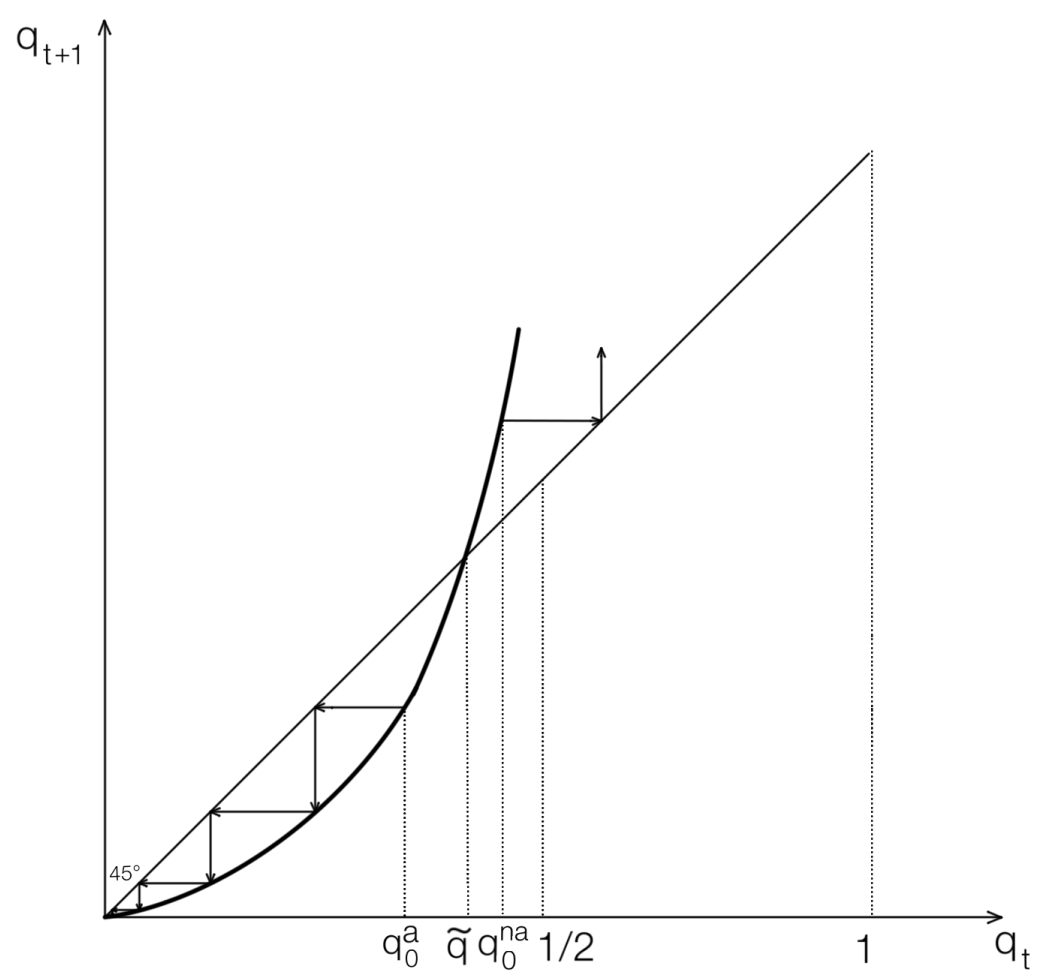

Figure 1a: Dynamics and Equilibria: Full Assimilation and Non-assimilation Equilibrium

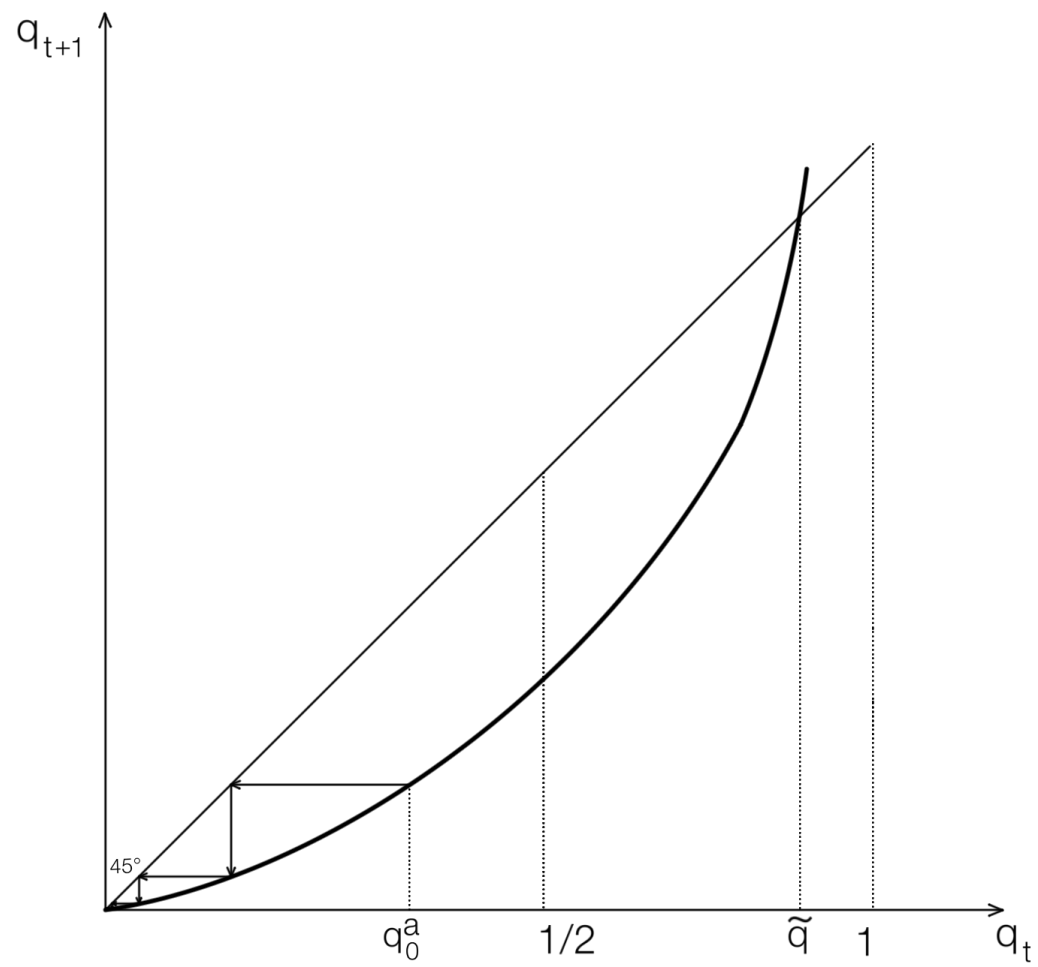

Figure 1b: Dynamics and Equilibria: Only Full Assimilation Equilibrium 


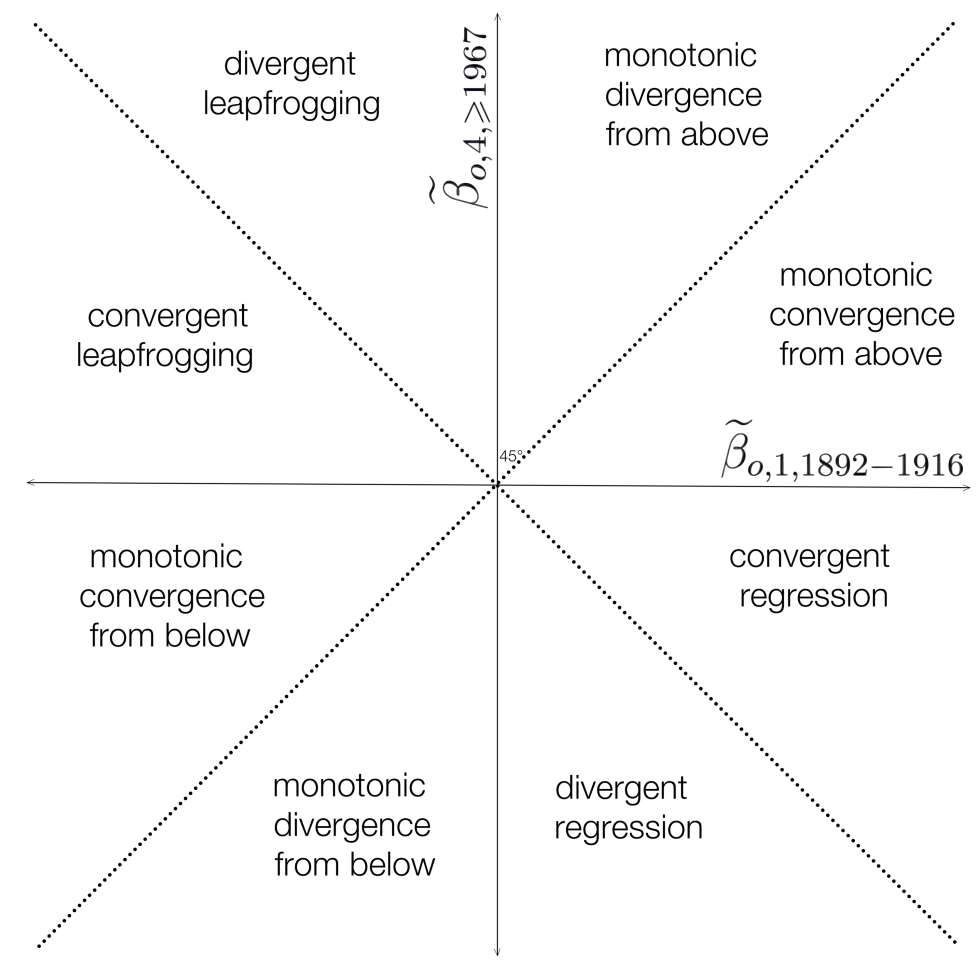

Figure 2a: Generational Convergence and Non-convergence Regions (by type)

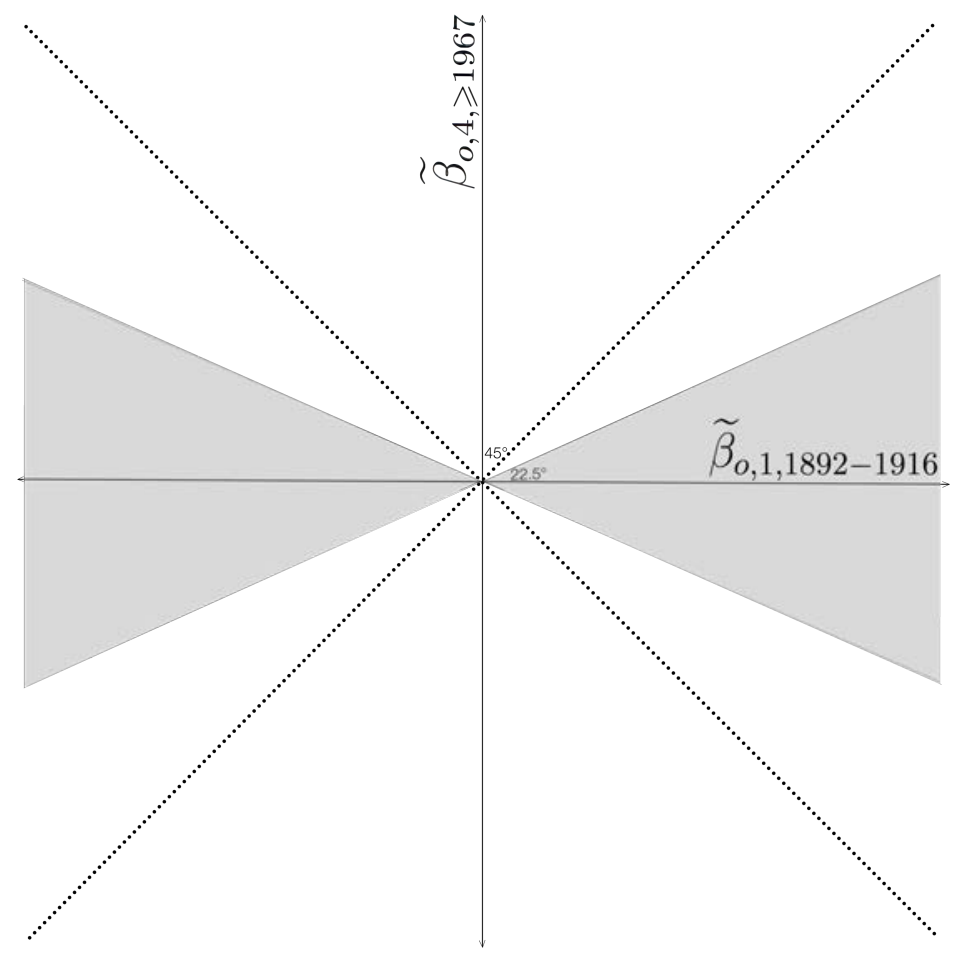

Figure 2b: Convergence Region Implied by the $22.5^{\circ}$ Cut-off Rule 


\section{References}

$[1]$

[2] Alba, Richard D. (1985). Italian Americans: Into the Twilight of Ethnicity. Prentice Hall, Englewood Cliffs.

[3] Albanesi, Stefania, and Claudia Olivetti (2016). "Gender roles and medical progress." Journal of Political Economy 124.3 650-695.

[4] Akerlof, George A. and Rachel E. Kranton (2000). "Economics and Identity." Quarterly Journal of Economics, 115(3): 715-753.

[5] Alesina, Alberto and Paola Giuliano (2010). "The Power of the Family," Journal of Economic Growth, June 2010, Vol 15, 93-125.

[6] Alesina, Alberto and Paola Giuliano (2011). "Preferences for Redistribution" in A. Bisin and J. Benhabib (eds.), Handbook of Social Economics, North Holland, 2011, Ch.4, 93-132.

[7] Alesina, Alberto and Paola Giuliano (2015). "Culture and institutions." Journal of Economic Literature 53.4: 898-944.

[8] Alesina, Alberto, Paola Giuliano, and Nathan Nunn (2013). "On the Origins of Gender Roles: Women and the Plough. Quarterly Journal of Economics, 128(2): 469-530.

[9] Alesina, Alberto and Nicola Fuchs-Schuendeln (2007). "Good bye Lenin (or not?): The Effect of Communism on People's Preferences. The American Economic Review 97(4): 1507-1528.

[10] Alesina, Alberto and George-Marios Angeletos (2005). "Fairness and Redistribution: US vs. Europe," American Economic Review, September 2005, 95: 913-35

[11] Alesina, Alberto and Edward Glazer (2004). "Fighting Poverty in the US and Europe: A World of Difference", Oxford University Press, Oxford UK.

[12] Algan, Yann and Pierre Cahuc (2007). "The Roots of Low European Employment: Family Culture?" in NBER International Seminar on Macroeconomics 2005, MIT Press, 65-109.

[13] Algan, Yann and Pierre Cahuc (2010). "Inherited Trust and Growth." American Economic Review, 100(5): 2060-2092.

[14] Algan, Yann, Pierre Cahuc, and Andrei Shleifer (2013). "Teaching Practices and Social Capital." American Economic Journal: Applied Economics, 5(3): 189-210.

[15] Algan, Yann, Alberto Bisin, Alan Manning, and Thierry Verdier (2012). Cultural Integration of Immigrants in Europe. Oxford University Press.

[16] Antecol, Heather (2000). "An Examination of Cross-Country Differences in the Gender Gap in Labor Force Participation Rates." Labour Economics, 7(4): 409-426.

[17] Benabou, Roland (2008). "Ideology", Journal of the European Economic Association, 6(2-3): 321-352 
[18] Benabou, Roland and Jean Tirole (2006). " Belief in a Just World and Redistributive Politics", Quarterly Journal of Economics, 121(20:699-746.

[19] Benabou, Roland and Jean Tirole (2016). "Mindful Economics: The Production, Consumption, and Value of Beliefs", Journal of Economic Perspectives: 30(3), Summer, 141-164.

[20] Bisin, Alberto and Thierry Verdier (2000). "Beyond the Melting Pot: Cultural Transmission and Dynamics of Preferences." Quarterly Journal of Economics, 115(3): 955-988.

[21] Bisin, Alberto and Thierry Verdier (2001). "The Economics of Cultural Transmission and the Evolution of Preferences." Journal of Economic Theory, 97(2): 298-319.

[22] Bisin, Aberto and Thierry Verdier (2010). "The Economics of Cultural Transmission and Socialization." Handbook of Social Economics, Elsevier

[23] Bisin, Alberto, Giorgio Topa, and Thierry Verdier (2004). "Cooperation as a Transmitted Cultural Trait." Rationality and Society 16: 477-507.

[24] Bisin, Alberto, Eleonora Pattachini, Thierry Verdier, and Yves Zenou (2011). "Formation and Persistence of Oppositional Identities." European Economic Review, 55(8): 1046-1071.

[25] Borjas, Geroge J. (1992). "Ethnic Capital and Intergenerational Mobility." Quarterly Journal of Economics, 107(1): 123-150.

[26] Borjas, George J. (1995). "Ethnicity, Neighborhoods, and Human-Capital Externalities." American Economic Review, 85(3): 365-390.

[27] Bowles, Samuel (1998). "Endogenous Preferences: The Cultural Consequences of Markets and Other Economic Institutions." Journal of Economic Literature, 36(1): 75-111.

[28] Boyd, Robert and Peter J. Richerson (1985). Culture and the Evolutionary Process. University of Chicago Press.

[29] Boyd, Robert and Peter J. Richerson (2005). The Origin and Evolution of Culture. Oxford University Press.

[30] Cavalli-Sforza, Luigi L. and Marcus W. Feldman (1981). Cultural Transmission and Evolution: A Quantitative Approach. Princeton University Press.

[31] Cavalli-Sforza, Luigi L. (2001). Genes, Peoples, and Languages. University of California Press.

[32] Di Tella, Rafael, Sebastian Galiani and Ernesto Schargrodsky (2007). "The Formation of Beliefs: Evidence from the Allocation of Land Titles." Quarterly Journal of Economics, 122(1): 209-241.

[33] Doepke, Matthias and Fabrizio Zilibotti (2008). "Occupational Choice and the Spirit of Capitalism." Quarterly Journal of Economics, 123(2): 747-793.

[34] Durante, Ruben (2009). "Risk, cooperation and the economic origins of social trust: an empirical investigation." MPRA Paper No. 25887. 
[35] Fehr, Ernst (2009). "On the Economics and Biology of Trust." Journal of the European Economic Association, 7(2-3): 235-266.

[36] Fernandez, Raquel (2007). "Women, Work and Culture." Journal of the European Economic Association, 5(2-3): 305-332.

[37] Fernandez, Raquel (2008). "Culture and Economics." in S.N Durlauf and L.E. Blume, New Plagrave Dictionary of Economics, 2nd edition, Palgrave Macmillan.

[38] Fernandez, Raquel (2011). "Does Culture Matter." in, J. Benhabib, M. O. Jackson and A. Bisin, Handbook of Social Economics, 1A, North-Holland, 481-510.

[39] Fernandez, Raquel (2013). "Culture change as Learning: The Evolution of Female Labor Force Participation over a Century" American Economic Review, 103(1): 472-500.

[40] Fernandez, Raquel and Alessandra Fogli (2006). "Fertility: the role of Culture and Family Experience." Journal of the European Economic Association, 4(2-3): 552-561.

[41] Fernandez, Raquel and Alessandra Fogli (2009), "Culture: An Empirical Investigation of Beliefs, Work and Fertility." American Economic Journal: Macroeconomics, 1(1): 146-177.

[42] Giavazzi, Francesco, Fabio Schiantarelli, and Michel Serafinelli (2013) "Culture, Policies and Labor Market outcomes." Journal of the European Economic Association, 11(6): 1256-1289.

[43] Giuliano, Paola (2007). "Living Arrangements in Western Europe: Does Cultural Origin Matter?" Journal of the European Economic Association, 5(5): 927-952.

[44] Giuliano, Paola and Antonio Spilimbergo (2014). "Growing up in a Recession." The Review of Economic Studies 81(2): 787-817.

[45] Goldin, Claudia (2006). "The Quiet Revolution that Transformed Women's Employment, Education, and Family." American Economic Review 96(2): 1-21.

[46] Greeley, Andrew M. (1974). "Ethnicity in the United States: A Preliminary Reconnaissance." John Wiley \& Sons.

[47] Gruber, Jonathan and Daniel M. Hungerman (2008). "The Church Versus the Mall: What Happens When Religion Faces Increased Secular Competition?" Quarterly Journal of Economics, 123(2): 831-862.

[48] Guiso, Luigi, Paola Sapienza, and Luigi Zingales (2006). "Does Culture Affect Economic Outcomes?" Journal of Economic Perspectives, 20(2): 23-48.

[49] Guiso, Luigi, Paola Sapienza, and Luigi Zingales (2008). "Alfred Marshall Lecture: Social Capital as Good Culture." Journal of the European Economic Association, 6(2-3): 295-320.

[50] Guiso, Luigi, Paola Sapienza, and Luigi Zingales (2013). "Long-Term Persistence." Journal of the European Economic Association, forthcoming

[51] Inglehart, Ronald and Wayne E. Baker (2000). "Modernization, Cultural Change, and the Persistence of Traditional Values." American Sociological review, 65(1): 19-51. 
[52] Konya, István (2005). "Minorities and Majorities: A Dynamic Model of Assimilation." Canadian Journal of Economics, 38(4): 1431-1452.

[53] Lazear, Edward P. (1999). "Culture and Language." Journal of Political Economy, 107(6): 95-126.

[54] Luttmer, Erzo F. P. and Monica Singhal (2011). "Culture, Context, and the Taste for Redistribution." American Economic Journal: Economic Policy, 3(1): 157-79.

[55] Muennig P, Johnson G, Kim J, Smith T, Rosen Z. (2011) "The General Social SurveyNational Death Index: an innovative new dataset for the social sciences." $B M C$ Research Notes. 2011; 4:385.

[56] Panebianco, Fabrizio. (2014) "Socialization networks and the transmission of interethnic attitudes." Journal of Economic Theory 150 (2014): 583-610.

[57] Pichler, Michael M. (2010) "The economics of cultural formation of preferences." No. 431. Working papers. Institute of Mathematical Economics.

[58] Piketty, Thomas (1995). "Social Mobility and Redistributive Politics", Quarterly Journal of Economics, 110(3); 551-584.

[59] Putnam, Robert D. (1993). "Making Democracy Work." Princeton University Press.

[60] Rice, Tom W. and Jan L. Feldman (1997). "Civic Culture and Democracy from Europe to America." The Journal of Politics, 59(4): 1143-1172.

[61] Roland, Gerard (2004). "Understanding Institutional Change: Fast-Moving and SlowMoving Institutions." Studies in Comparative International Development, 38(4): 109-131.

[62] Tabellini, Guido (2008a). "Presidential Address: Institutions and Culture." Journal of the European Economic Association Papers and Proceedings, 6(2-3): 255-294.

[63] Tabellini, Guido (2008b). "The Scope of Cooperation: Values and Incentives." The Quarterly Journal of Economics, 123(3): 905-950.

[64] Tabellini, Guido (2010). "Culture and Institutions: Economic Development in the Regions of Europe." Journal of the European Economic Association, 8(4): 677-716.

[65] Vaughan, Daniel (2013). "Nurture vs. nurture: Endogenous parental and peer effects and the transmission of culture." (No. 2013-04). Working Papers, Banco de México. 


\section{Appendix 1: Robustness (For Online Publication Only)}

Table A1: Sensitivity of Convergence Across Different Criteria

\begin{tabular}{|c|c|c|c|c|c|}
\hline & & Gen $4 \pi_{45}$ & Gen $4 \pi_{33}$ & Gen $4 \pi_{22.5}$ & Gen $4 \pi_{15}$ \\
\hline Group A - Cooperation & $\begin{array}{l}\text { trust } \\
\text { fair } \\
\text { helpful }\end{array}$ & $86 \%$ & $86 \%$ & $81 \%$ & $67 \%$ \\
\hline Group B - Government & $\begin{array}{l}\text { eqwlth } \\
\text { helppoor } \\
\text { polviews }\end{array}$ & $76 \%$ & $52 \%$ & $38 \%$ & $38 \%$ \\
\hline Group C - Religion & $\begin{array}{l}\text { attend } \\
\text { pray } \\
\text { reliten } \\
\text { postlife } \\
\text { prayer }\end{array}$ & $77 \%$ & $69 \%$ & $60 \%$ & $40 \%$ \\
\hline Group D - Family & $\begin{array}{l}\text { thnkself } \\
\text { obey } \\
\text { pillok } \\
\text { aged } \\
\text { divlaw } \\
\text { socrel }\end{array}$ & $86 \%$ & $69 \%$ & $67 \%$ & $50 \%$ \\
\hline Group E - Gender Roles & $\begin{array}{l}\text { fechld } \\
\text { fepol }\end{array}$ & $71 \%$ & $71 \%$ & $64 \%$ & $64 \%$ \\
\hline Group F - Abortion & $\begin{array}{l}\text { abany } \\
\text { abrisk }\end{array}$ & $71 \%$ & $57 \%$ & $57 \%$ & $50 \%$ \\
\hline Group G - Sexual Behavior & $\begin{array}{l}\text { premarsx } \\
\text { homosex }\end{array}$ & $71 \%$ & $50 \%$ & $43 \%$ & $36 \%$ \\
\hline Group H - Mobility/Success & getahead & $71 \%$ & $57 \%$ & $57 \%$ & $14 \%$ \\
\hline & & \multicolumn{4}{|c|}{ Rank Correlation } \\
\hline & & Gen $4 \pi_{45}$ & Gen $4 \pi_{33}$ & Gen $4 \pi_{22.5}$ & Gen $4 \pi_{15}$ \\
\hline & $\begin{array}{l}\text { Gen } 4 \pi_{45} \\
\text { Gen } 4 \pi_{33} \\
\text { Gen } 4 \pi_{22.5} \\
\text { Gen } 4 \pi_{15}\end{array}$ & $\begin{array}{l}1.00 \\
0.59 \\
0.54 \\
0.43\end{array}$ & $\begin{array}{l}1.00 \\
0.88 \\
0.76\end{array}$ & $\begin{array}{l}1.00 \\
0.72\end{array}$ & 1.00 \\
\hline
\end{tabular}

Notes: The table shows different orderings of the speed of convergence according to the percentage of country-wave observations for which the absolute value of the deviation from the norm in the first generation has been cut by any amount (Gen $\left.4 \pi_{45}\right)$, by a third (Gen 4 $\pi_{30}$ ), by half (Gen $4 \pi_{22.5}$ ), and by two thirds $\left(\right.$ Gen $4 \pi_{15}$ ) by generation 4 . The second table lists the rank correlations between the different convergence criteria. 
Table A2: Limited Controls: Convergence of Cultural Attitudes (by Groups), Comparing Generation 4 and 2

\begin{tabular}{|c|c|c|c|c|c|c|}
\hline & & Gen $4 \pi_{22.5}$ & Gen $2 \pi_{22.5}$ & $\triangle$ & $90 \% \mathrm{CI}$ & $95 \% \mathrm{CI}$ \\
\hline Group A - Cooperation & $\begin{array}{l}\text { trust } \\
\text { fair } \\
\text { helpful }\end{array}$ & $81 \%$ & $14 \%$ & $67 \%$ & $(19 \%, 52 \%)$ & $(14 \%, 57 \%)$ \\
\hline Group B - Government & \begin{tabular}{|l|} 
eqwlth \\
helppoor \\
polviews
\end{tabular} & $52 \%$ & $67 \%$ & $-14 \%$ & $(5 \%, 40 \%)$ & $(0 \%, 48 \%)$ \\
\hline Group C - Religion & \begin{tabular}{|l} 
attend \\
pray \\
reliten \\
postlife \\
prayer
\end{tabular} & $57 \%$ & $43 \%$ & $14 \%$ & $(0 \%, 29 \%)$ & $(-3 \%, 31 \%)$ \\
\hline Group D - Family & \begin{tabular}{|l} 
thnkself \\
obey \\
pillok \\
aged \\
divlaw \\
socrel
\end{tabular} & $64 \%$ & $24 \%$ & $40 \%$ & $(10 \%, 43 \%)$ & $(14 \%, 40 \%)$ \\
\hline Group E - Gender Roles & $\begin{array}{l}\text { fechld } \\
\text { fepol }\end{array}$ & $79 \%$ & $50 \%$ & $29 \%$ & $(0 \%, 43 \%)$ & $(-7 \%, 50 \%)$ \\
\hline Group F - Abortion & $\begin{array}{l}\text { abany } \\
\text { abrisk }\end{array}$ & $79 \%$ & $79 \%$ & $0 \%$ & $(-14 \%, 29 \%)$ & $(-14 \%, 29 \%)$ \\
\hline Group G - Sexual Behavior & $\begin{array}{l}\text { premarsx } \\
\text { homosex }\end{array}$ & $71 \%$ & $50 \%$ & $21 \%$ & $(-7 \%, 50 \%)$ & $(-7 \%, 57 \%)$ \\
\hline Group H - Mobility/Success & getahead & $57 \%$ & $57 \%$ & $0 \%$ & $(-29 \%, 43 \%)$ & $(-29 \%, 43 \%)$ \\
\hline
\end{tabular}


Table A3: GB Benchmark: Convergence of Cultural Attitudes (by Groups), Comparing Generation 4 and 2

\begin{tabular}{|c|c|c|c|c|c|c|}
\hline & & Gen $4 \pi_{22.5}$ & Gen $2 \pi_{22.5}$ & $\triangle$ & $90 \% \mathrm{CI}$ & $95 \% \mathrm{CI}$ \\
\hline Group A - Cooperation & $\begin{array}{l}\text { trust } \\
\text { fair } \\
\text { helpful }\end{array}$ & $78 \%$ & $28 \%$ & $50 \%$ & $(14 \%, 48 \%)$ & $(14 \%, 57 \%)$ \\
\hline Group B - Government & $\begin{array}{l}\text { eqwlth } \\
\text { helppoor } \\
\text { polviews }\end{array}$ & $50 \%$ & $39 \%$ & $11 \%$ & $(10 \%, 43 \%)$ & $(5 \%, 48 \%)$ \\
\hline Group C - Religion & $\begin{array}{l}\text { attend } \\
\text { pray } \\
\text { reliten } \\
\text { postlife } \\
\text { prayer }\end{array}$ & $57 \%$ & $43 \%$ & $13 \%$ & $(0 \%, 31 \%)$ & $(-3 \%, 34 \%)$ \\
\hline Group D - Family & $\begin{array}{l}\text { thnkself } \\
\text { obey } \\
\text { pillok } \\
\text { aged } \\
\text { divlaw } \\
\text { socrel }\end{array}$ & $64 \%$ & $25 \%$ & $39 \%$ & $(12 \%, 38 \%)$ & $(10 \%, 40 \%)$ \\
\hline Group E - Gender Roles & $\begin{array}{l}\text { fechld } \\
\text { fepol }\end{array}$ & $75 \%$ & $75 \%$ & $0 \%$ & $(-7 \%, 43 \%)$ & $(-7 \%, 43 \%)$ \\
\hline Group F - Abortion & $\begin{array}{l}\text { abany } \\
\text { abrisk }\end{array}$ & $58 \%$ & $42 \%$ & $17 \%$ & $(-7 \%, 36 \%)$ & $(-14 \%, 43 \%)$ \\
\hline Group G - Sexual Behavior & $\begin{array}{l}\text { premarsx } \\
\text { homosex }\end{array}$ & $58 \%$ & $42 \%$ & $17 \%$ & $(0 \%, 43 \%)$ & $(-7 \%, 50 \%)$ \\
\hline Group H - Mobility/Success & getahead & $33 \%$ & $50 \%$ & $-17 \%$ & $(-29 \%, 43 \%)$ & $(-29 \%, 43 \%)$ \\
\hline
\end{tabular}


Table A4: Including Mexico in Benchmark: Convergence of Cultural Attitudes (by Groups), Comparing Generation 4 and 2

\begin{tabular}{l|l|ccccc}
\hline & & Gen $4 \pi_{22.5}$ & Gen $2 \pi_{22.5}$ & $\triangle$ & $90 \%$ CI & $95 \%$ CI \\
\hline Group A - Cooperation & $\begin{array}{l}\text { trust } \\
\text { fair } \\
\text { helpful }\end{array}$ & $71 \%$ & $33 \%$ & $38 \%$ & $(14 \%, 48 \%)$ & $(10 \%, 52 \%)$ \\
\hline Group B - Government & $\begin{array}{l}\text { eqwlth } \\
\text { helppoor } \\
\text { polviews }\end{array}$ & $43 \%$ & $43 \%$ & $0 \%$ & $(0 \%, 43 \%)$ & $(-5 \%, 43 \%)$ \\
\hline Group C - Religion & $\begin{array}{l}\text { attend } \\
\text { pray } \\
\text { reliten } \\
\text { postlife } \\
\text { prayer }\end{array}$ & $57 \%$ & $46 \%$ & $11 \%$ & $(3 \%, 31 \%)$ & $(0 \%, 34 \%)$ \\
\hline Group D - Family & $\begin{array}{l}\text { thnkself } \\
\text { obey } \\
\text { pillok } \\
\text { aged } \\
\text { divlaw } \\
\text { socrel }\end{array}$ & $67 \%$ & $43 \%$ & $24 \%$ & $(10 \%, 36 \%)$ & $(7 \%, 40 \%)$ \\
\hline Group E - Gender Roles & $\begin{array}{l}\text { fechld } \\
\text { fepol }\end{array}$ & $71 \%$ & $43 \%$ & $29 \%$ & $0 \%, 43 \%)$ & $(-7 \%, 50 \%)$ \\
\hline Group F - Abortion & $\begin{array}{l}\text { abany } \\
\text { abrisk }\end{array}$ & $64 \%$ & $50 \%$ & $14 \%$ & $(-7 \%, 36 \%)$ & $(-14 \%, 43 \%)$ \\
\hline Group G - Sexual Behavior H - Mobility/Success & $\begin{array}{l}\text { premarsx } \\
\text { homosex }\end{array}$ & $43 \%$ & $43 \%$ & $0 \%$ & $(0 \%, 43 \%)$ & $(-7 \%, 50 \%)$ \\
\hline getahead & $57 \%$ & $71 \%$ & $-14 \%$ & $(-29 \%, 43 \%)$ & $(-29 \%, 43 \%)$ \\
\hline
\end{tabular}

Notes: This table replicates Table 4 using a benchmark that includes the attitudes of Mexican immigrants. Gen $4 \pi_{22.5}$ (Gen $2 \pi_{22.5}$ ) denotes the average percentage of country observations for which the absolute value of the deviation from the norm has been cut at least in half between generation 1 and generation 4 (2) within each group.

Table A5: List of Matched Attitudes between the General Social Survey (GSS) and the European Values Survey/World Values Survey (EVS/WVS)

\begin{tabular}{|l|l|l|}
\hline GSS & $\begin{array}{l}\text { EVS/WVS Question } \\
\text { Number }\end{array}$ & Description of EVS variable \\
\hline trust & a165 & Most people can be trusted ( $\mathrm{y}=1$ for yes if $\left.x_{E V S}=1\right)$ \\
attend & $\mathrm{f} 028$ & How often do you attend religious services $\left(\mathrm{y}=1\right.$ for less often if $\left.x_{E V S}>3\right)$ \\
pray & f063 & How important is God in your life $\left(\mathrm{y}=1\right.$ for less important if $\left.x_{E V S}<7\right)$ \\
postlife & $\mathrm{f} 051$ & Believe in life after death $\left(\mathrm{y}=1\right.$ for no if $\left.x_{E V S}=0\right)$ \\
thnkself & $\mathrm{a} 029$ & Important child qualities: independence $\left(\mathrm{y}=1\right.$ for important if $\left.x_{E V S}=1\right)$ \\
obey & $\mathrm{a} 042$ & Important child qualities: obedience $\left(\mathrm{y}=1\right.$ for not important if $\left.x_{E V S}=0\right)$ \\
fechild & $\mathrm{d} 061$ & Pre-school child suffers with working mother $\left(\mathrm{y}=1\right.$ for yes if $\left.x_{E V S}>2\right)$ \\
abany & $\mathrm{f} 120$ & Justifiable: abortion $\left(\mathrm{y}=1\right.$ for yes if $\left.x_{E V S}=10\right)$ \\
homosex & $\mathrm{f} 118$ & Justifiable: homosexuality $\left(\mathrm{y}=1\right.$ for yes if $\left.x_{E V S}>7\right)$ \\
\hline
\end{tabular}

Notes: The responses from the EVS/WVS have been recoded to have a binary outcome. We indicate the correspondence between GSS and EVS/WVS and the original value(s) from the EVS/WVS that are matched with the recoded GSS variables. $y$ denotes the indicator variable in the first stage Probit. $x_{E V S}$ denotes the answer number to the EVS/WVS questions. 


\section{Appendix 2: Phase Diagram and Location of $\tilde{q}$ (For On- line Publication Only)}

Re-writing equation (8) in the text, the dynamics of assimilation is determined by:

$$
\begin{aligned}
& q_{t+1}=\left(1-\frac{\left(1-q_{t}\right) \theta^{M} V-q_{t} \theta^{m} V-d\left(\frac{\varphi_{0}-\beta\left[\left(1-q_{t}\right) \theta^{M} V-q_{t} \theta^{m} V-\underline{z}\right]}{\frac{c(\bar{z}-\underline{z})}{d}-\beta d}\right)-\underline{z}}{\bar{z}-\underline{z}}\right) q_{t} \\
& \frac{d q_{t+1}}{d q_{t}}=\left(1-\frac{\left(1-q_{t}\right) \theta^{M} V-q_{t} \theta^{m} V-d\left(\frac{\varphi_{0}-\beta\left[\left(1-q_{t}\right) \theta^{M} V-q_{t} \theta^{m} V-\underline{z}\right]}{\frac{c(\bar{z}-\underline{z})}{d}-\beta d}\right)-\underline{z}}{\bar{z}-\underline{z}}\right)+\left(\frac{\theta^{M} V+\theta^{m} V+\beta d \frac{\left[\theta^{M} V-q_{t} \theta^{m} V\right]}{\frac{c(\bar{z}-z)}{d}-\beta d}}{\bar{z}-\underline{z}} t\right) q_{t}>0 \\
& \frac{d^{2} q_{t+1}}{d q_{t}^{2}}=\frac{2 c\left(\theta^{M} V+\theta^{m} V\right)}{c(\bar{z}-\underline{z})-\beta d^{2}}>0
\end{aligned}
$$

Therefore the relationship between $q_{t+1}$ and $q_{t}$ (the phase line) starts at zero and it is increasing and convex. It intersects the 45 degree line also at $\tilde{q}$, where $\tilde{q}$ satisfies $(1-\tilde{q}) \theta^{M} V-$ $\tilde{q} V \theta^{m} V-d \tau^{*}=\underline{z}$, so that there are no gain from assimilation and $G\left((1-\tilde{q}) \theta^{M} V-\tilde{q} \theta^{m} V-d \tau^{*}\right)=$ 0. Our parametrization implies:

$$
\tilde{q}=\frac{\theta^{M} V-\frac{\varphi_{0} d^{2}}{c(\bar{z}-\underline{z})}-\underline{z}}{\theta^{M} V+\theta^{m} V}
$$

The numerator of the first line on the right hand side of (A4) is strictly positive, because we assume that $(1-q) \theta^{M} V-q \theta^{m} V-d \tau^{*} \geq \underline{z}$ which implies that $(1-q) \theta^{M} V-q \theta^{m} V-\frac{\varphi_{0} d^{2}}{c(\bar{z}-\underline{z})}-\underline{z} \geq 0$. Hence $\tilde{q}>0$. The numerator and denominator of (A4) also imply that $\tilde{q}<1$. Therefore, $0<\tilde{q}<1$ as claimed in the text. 